Check for updates

Cite this: RSC Adv., 2017, 7, 34830

\title{
Rational design of doubly-bridged chromophores for singlet fission and triplet-triplet annihilation $\uparrow$
}

\author{
S. Ito, (D) ${ }^{a}$ T. Nagami ${ }^{a}$ and M. Nakano (D) *ab
}

We demonstrate rational designs of excitation energies and electronic couplings using doubly-bridged chromophores for exciton down- and up-conversions, the former and latter of which are known as singlet fission and triplet-triplet annihilation, respectively. We deduce energetic conditions suitable for these two conversion processes based on quantum interferences within a bridge as well as between two bridges. The idea is at first proposed at the Hückel approximation level of theory in a theoretical model, and then, is realized for molecular systems of polyyne bridges with several lengths of ethynyl units as well as with different linked sites by ab initio quantum chemistry calculation. The result is analyzed in detail by decomposing the electronic couplings into direct-overlap and bridge-mediated couplings from each bridge, which definitely confirms the quantum interference between the bridges. Further analysis using perturbation theory clarifies this effect on the energetics concerning singlet fission and triplettriplet annihilation. Estimation of the singlet fission time constants for the molecules designed to have exothermic singlet fission gave $10^{2}-10^{4} \mathrm{ps}$, which is much faster than for most tetracene dimers reported previously. The present study provides a widely applicable molecular design guideline for tuning the energetic conditions by selective control of the electronic coupling matrix elements, which can be systematically achieved by considering the relative phases and distributions of the $\pi$-orbitals in chromophores and bridges.

Received 30th May 2017

Accepted 4th July 2017

DOI: $10.1039 / \mathrm{c} 7 \mathrm{ra06032g}$

rsc.li/rsc-advances

\section{Introduction}

Rational design of opto-electronic materials is indispensable for efficient use of the photon energy of sunlight for realizing a sustainable society in the future. Photovoltaic cells are an important class of devices that convert the photon energy into electricity. Unfortunately, most chromophores cannot use the whole range of the solar spectrum. In order to use a wider range of the solar spectrum in photovoltaic cells, potential excited state processes called singlet fission $(\mathrm{SF})^{\mathbf{1 , 2}}$ and triplet-triplet annihilation (TTA) $)^{3,4}$ are widely investigated. In SF, a singlet exciton splits into a pair of triplet excitons with a lower energy, while in TTA, a pair of triplet excitons gets together into a singlet exciton with a higher energy. The former is a photon down-conversion process, while the latter an up-conversion

${ }^{a}$ Department of Materials Engineering Science, Graduate School of Engineering Science, Osaka University, Toyonaka, Osaka 560-8531, Japan. E-mail: mnaka@ cheng.es.osaka-u.ac.jp

${ }^{b}$ Center for Spintronics Research Network (CSRN), Graduate School of Engineering Science, Osaka University, Toyonaka, Osaka 560-8531, Japan

$\dagger$ Electronic supplementary information (ESI) available: Other possible phase patterns for case (I-PP); definition of relative phase of molecular orbital; CT state energy dependence; transition density in tetracene; decomposition analysis of electronic coupling for BET-B; proof of eqn (16); eigenvalues and eigenvectors of Hamiltonian eqn (1): detailed decomposition analysis of 1-(1,1). See DOI: $10.1039 / \mathrm{c} 7 \mathrm{ra06032g}$ process. TTA is also applicable for highly efficient fluorescent materials that emit blue light by using red light.

The substantially important requirements for SF and TTA are the energy level matching conditions. The former requires the energetic driving force, $E\left(\mathrm{~S}_{1}\right)>2 E(\mathrm{~T})$, while the latter does, $E\left(\mathrm{~S}_{1}\right)$ $<2 E(\mathrm{~T})$, where $E\left(\mathrm{~S}_{1}\right)$ and $E(\mathrm{~T})$ are the lowest singlet and triplet excitation energies, respectively. ${ }^{\mathbf{1 , 3 - 1 2}}$ Indeed, on the basis of these energetic conditions, in SF research, tetracene, ${ }^{\mathbf{1 3 - 2 5}}$ pentacene ${ }^{19-21,25-33}$ and their derivatives are the most investigated, while in TTA research, anthracene derivatives, rubrene and perylene are well investigated. ${ }^{3,4}$ In addition to the energetic requirements, both conversion processes need sufficient interchromophore interaction described by $\pi$-orbital overlap, called electronic coupling. ${ }^{\mathbf{1 , 2}}$ The electronic coupling relevant to SF and TTA can be controlled by changing the crystal structure through chemical modifications. ${ }^{15,19,22,34-40}$ As another way of controlling electronic coupling, the use of covalently-linked systems is promising because the electronic coupling can be designed at the molecular level. ${ }^{41-57}$ In the previous study, we proposed several ways of tuning electronic coupling concerning SF based on the quantum interference. ${ }^{52}$ We observed that covalently-linked systems with constructive quantum interference induce faster SF than those with destructive quantum interference. Besides, we found similar amplitudes of the electronic coupling matrix elements originating from the bridgemediated contributions. 
On the other hand, using perturbation analysis, relative values of these electronic coupling matrix elements are shown to have a strong impact on the relative energy of singlet and triplet-pair excitons, and thus on the yield of triplet-pair exciton in SF. ${ }^{58}$ This indicates the energy level matching and electronic coupling are deeply connected and thus cannot be considered separately. These results are expected to be useful also for designing TTA materials because a similar electronic Hamiltonian can be considered in both SF and TTA. Therefore, the element-selective electronic coupling design is desired for realizing future high-performance electronic devices using SF and/or TTA. The element-selective electronic coupling design is not a simple task from a material design viewpoint. In singlybridged systems on which previous researches focused, we showed that all the Fock matrix elements (electronic couplings) are similar in their amplitude at least when the chromophores have negligible direct overlap. ${ }^{52}$ This is because the any bridgemediated couplings have the same prefactor in a singly-bridged chromophore. Therefore, we have to consider another strategy to achieve this.

In this study, we propose a novel materials design strategy for SF and TTA, that is, double bridging of chromophores. In doubly-bridged systems, as will be shown in this study, the bridge-mediated electronic couplings are represented by the sum of each bridge contributions. This extends the design possibility to realize "element-selective tuning of electronic couplings". And doubly-bridge chromophores can be designed so as to satisfy the energy balance suitable for either SF or TTA. We aim at constructing such design guidelines by combining the quantum-interference-based electronic-coupling control and by using perturbation analysis of its influence on the energetics in SF and TTA. The paper is organized as follows. Section 2 explains the five states model for SF and TTA together with its perturbation analysis. We also summarize the previous results and provide a strategy based on the quantum interference in covalently-linked systems for tuning the electronic couplings between chromophores. In Section 3, the model system is briefly described. In Section 4, we explain the computational details of $a b$ initio quantum chemistry calculations for determining the energy levels of the chromophore. In Section 5 , the relationship between the odd-even parity of the bridge and its linked-position dependence is clarified. The energetics is also analysed by using perturbation theory. Comparison of the present results with an experimental result is addressed. The relationship between the present result in $\mathrm{SF} /$ TTA and the previous studies of electronic coupling in other phenomena such as intramolecular electron/hole transfer is also discussed to illuminate the peculiar quantum interference in SF and TTA. The conclusion is given in Section 6.

\section{Theory}

\subsection{Smith and Michl's diabatic model for SF and TTA}

We consider the electronic Hamiltonian of a chromophore dimer described by the five diabatic excited states concerning SF and TTA, the space of which is spanned by the two local excitons (Frenkel exciton, FE), two charge-transfer (CT) excitons (cation-anion pair, CA, and anion-cation pair, AC) and a tripletpair (TT) exciton. ${ }^{\mathbf{1 , 2}}$

$$
\begin{gathered}
\left\langle\mathrm{S}_{1} \mathrm{~S}_{0}\right| \\
\left\langle\mathrm{S}_{0} \mathrm{~S}_{1}\right| \\
\langle\mathrm{CA}| \\
\langle\mathrm{AC}| \\
\langle\mathrm{TT}|
\end{gathered}\left(\begin{array}{ccccc}
E_{\mathrm{FE}} & & & & \\
J_{\mathrm{ex}} & E_{\mathrm{FE}} & & & \\
E_{\mathrm{LL}} & -F_{\mathrm{HH}} & E_{\mathrm{CT}} & & \\
-F_{\mathrm{HH}} & F_{\mathrm{LL}} & 0 & E_{\mathrm{CT}} & \\
V_{2 \mathrm{e}} & V_{2 \mathrm{e}}^{\prime} & \sqrt{\frac{3}{2}} F_{\mathrm{LH}} & \sqrt{\frac{3}{2}} F_{\mathrm{HL}} & E_{\mathrm{TT}}
\end{array}\right)
$$

here, $E_{\mathrm{X}}(\mathrm{X}=\mathrm{FE}, \mathrm{CT}$ and TT $)$ is the energy of the excited state $\mathrm{X}$; $J_{\text {ex }}$ is the exciton-exciton coupling between two Frenkel excitons; $F_{i j}(i, j=\mathrm{H}$ and $\mathrm{L})$ is the Fock coupling matrix between two MOs in neighbouring chromophores; $\mathrm{H}$ and $\mathrm{L}$ indicate the highest occupied molecular orbital (HOMO) and the lowest unoccupied MO (LUMO) of a chromophore, respectively; $V_{2 \mathrm{e}}$ and $V_{2 \mathrm{e}}^{\prime}$ represent the two-electron (direct) couplings between FE and TT states. In eqn (1), we ignore the two-electron couplings $V_{2 \mathrm{e}}$ and $V_{2 \mathrm{e}}^{\prime}$ since they are known to be negligibly small compared to the one-electron contributions. ${ }^{2}$ Also, the exchange contribution $\left(J_{\text {exch }}\right)$ in the exciton-exciton coupling, $J_{\text {ex }}$ $\left(=J_{\text {Coul }}+J_{\text {exch }}\right)$, is ignored since $J_{\text {exch }}$ is usually smaller than the Coulomb contribution $\left(J_{\text {Coul }}\right) \cdot{ }^{59}$ Unless we explicitly mention to it, the term "state" is used for FE, CT and TT diabatic bases, that is, electronic configurations.

Assuming the two CT states have much higher energies than the other three states, the effective Hamiltonian, which describes low-lying states primarily composed of the FE and TT states, is approximately expressed using quasi-degenerate perturbation theory as $\mathrm{s}^{2,58,60-62}$

$$
\begin{aligned}
& \begin{array}{l}
\left\langle\mathrm{S}_{1} \mathrm{~S}_{0}^{\prime}\right| \\
\left\langle\mathrm{S}_{0} \mathrm{~S}_{1}^{\prime}\right|
\end{array}\left(\begin{array}{ccc}
E_{\mathrm{FE}}^{\text {eff }} & \\
J^{\text {eff }} & E_{\mathrm{FE}}^{\text {eff }} & \\
V_{\mathrm{FE}-\mathrm{TT}}^{\mathrm{eff}} & V_{\mathrm{FE}-\mathrm{TT}^{\prime}}^{\text {eff }} & E_{\mathrm{TT}}^{\text {eff }}
\end{array}\right)= \\
&\left(\begin{array}{ccc}
E_{\mathrm{FE}}+\Delta E_{\mathrm{FE}} & \\
J_{\mathrm{Coul}}+J_{\mathrm{CT}} & E_{\mathrm{FE}}+\Delta E_{\mathrm{FE}} & \\
V_{\mathrm{FE}-\mathrm{TT}} & V_{\mathrm{FE}-\mathrm{TT}^{\prime}} & E_{\mathrm{TT}}+\Delta E_{\mathrm{TT}}
\end{array}\right)
\end{aligned}
$$

where the second-order contributions in the energies and couplings are given by,

$$
\begin{gathered}
V_{\mathrm{FE}-\mathrm{TT}}=\frac{1}{2}\left(\sqrt{\frac{3}{2}} \frac{-F_{\mathrm{HH}} F_{\mathrm{HL}}+F_{\mathrm{LL}} F_{\mathrm{LH}}}{E_{\mathrm{FE}}-E_{\mathrm{CT}}}+(\mathrm{FE} \leftrightarrow \mathrm{TT})\right) \\
V_{\mathrm{FE}-\mathrm{TT}^{\prime}}=\frac{1}{2}\left(\sqrt{\frac{3}{2}} \frac{-F_{\mathrm{HH}} F_{\mathrm{LH}}+F_{\mathrm{LL}} F_{\mathrm{HL}}}{E_{\mathrm{FE}}-E_{\mathrm{CT}}}+(\mathrm{FE} \leftrightarrow \mathrm{TT})\right) \\
\Delta E_{\mathrm{FE}}=\frac{F_{\mathrm{HH}}^{2}}{E_{\mathrm{FE}}-E_{\mathrm{CT}}}+\frac{F_{\mathrm{LL}}{ }^{2}}{E_{\mathrm{FE}}-E_{\mathrm{CT}}} \\
\Delta E_{\mathrm{TT}}=\frac{\left(\sqrt{3 / 2} F_{\mathrm{LH}}\right)^{2}}{E_{\mathrm{TT}}-E_{\mathrm{CT}}}+\frac{\left(\sqrt{3 / 2} F_{\mathrm{HL}}\right)^{2}}{E_{\mathrm{TT}}-E_{\mathrm{CT}}} \\
J_{\mathrm{CT}}=2 \frac{-F_{\mathrm{HH}} F_{\mathrm{LL}}}{E_{\mathrm{FE}}-E_{\mathrm{CT}}}
\end{gathered}
$$


here, (FE $\leftrightarrow \mathrm{TT}$ ) indicates the same term as the former except that the denominator is replaced by $E_{\mathrm{TT}}-E_{\mathrm{CT}}$. The energy correction $\Delta E_{\mathrm{FE}}$ and CT-mediated excitonic coupling $J_{\mathrm{CT}}$ were investigated in the analysis of absorption spectrum and exciton migration in the spectroscopic feature of $\pi$-conjugated molecular aggregates. ${ }^{60-62}$ The energy correction on TT state, $\Delta E_{\mathrm{TT}}$, was considered in the previous study on $\mathrm{SF}^{58}$ The couplings $V_{\mathrm{FE}-\mathrm{TT}}$ and $V_{\mathrm{FE}-\mathrm{TT}^{\prime}}$ represent one-electron contributions to the electronic coupling concerning SF and maybe TTA, and

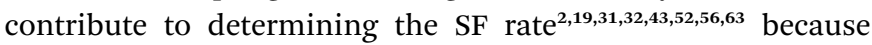
they correspond to the off-diagonal part connecting the FE and TT states.

In order to estimate an approximate energy balance between the FE and TT states after the perturbation through the CT states, we defined the effective energy difference between them, ${ }^{\mathbf{5 8}}$

$$
\left(E_{\mathrm{TT}}-E_{\mathrm{FE}}\right)^{\mathrm{eff}}=\left(E_{\mathrm{TT}}+\Delta E_{\mathrm{TT}}\right)-\left(E_{\mathrm{FE}}+\Delta E_{\mathrm{FE}}-\left|J^{\mathrm{eff}}\right|\right)
$$

A step-by-step view of this energy change by the perturbation through the CT states is shown in Fig. 1, where the effects of the energy corrections $\Delta E_{\mathrm{FE}}$ and $\Delta E_{\mathrm{TT}}$, together with the excitonexciton splitting $J^{\text {eff }}$, related to Davydov splitting, are shown. The effective energy difference would represent whether the molecule could exhibit SF or TTA as an exothermic process. In Fig. 1, we show four cases in this study: strong mixing with the CT states both in FE and TT states (Fig. 1a), weak mixing with the CT states both in FE and TT states (Fig. 1b), strong mixing with the CT states in FE but not in TT (Fig. 1c), and strong

(a)

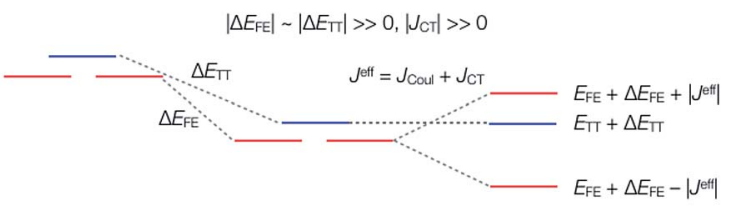

(b)

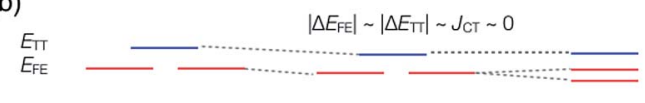

(c)

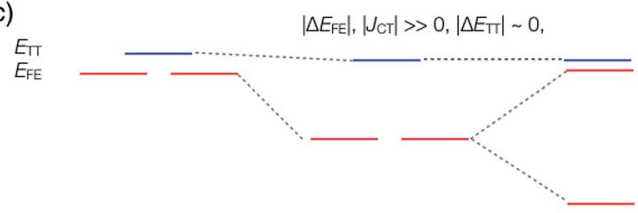

(d) $\left|\Delta E_{\mathrm{FE}}\right|,\left|J_{\mathrm{CT}}\right| \sim 0,\left|\Delta E_{T \mid}\right|>0$

$E_{\mathrm{TT}}$
$E_{\mathrm{FE}}$

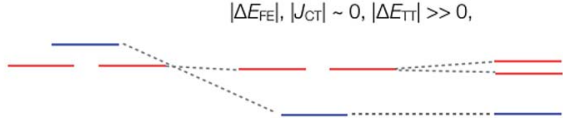

Fig. 1 Energy diagram of singlet (FE) and triplet-pair (TT) states through charge-transfer (CT) states mixing. The case (I-PP), where the CT states strongly mix with both the FE and TT states, (a); the case (I$\mathrm{PN}$ ), where the CT states barely mix with both the FE and TT states, (b); the case (II-PP), where the CT states strongly mix with the FE states only, (c); the case (II-PN), where the CT states strongly mix with the TT state only, (d). See Section 2.3 for detail. mixing with the CT states in TT but not in FE (Fig. 1d). Note that eqn (8) is not the eigenvalue difference itself obtained from this effective Hamiltonian (eqn (2)) but should give the approximate relative energy balance between the FE-dominated and TTdominated lowest excited eigenstates. Although Davydov splitting, which corresponds to $2\left|J^{\mathrm{eff}}\right|$ in this dimer model, is widely used as a measure of the electronic coupling between chromophores, this does not give sufficient information for judging the possibility for SF and TTA. For SF and TTA, we need to know the non-horizontal couplings $\left(F_{\mathrm{HL}}\right.$ and $\left.F_{\mathrm{LH}}\right)$ that may be difficult to be extracted from UV/vis absorption spectrum. Also, we have to be careful that the strong electronic coupling estimated from only Davydov splitting might not indicate favourable situation for SF or TTA. Although the low-lying excited absorption bands actually includes the mixing of FE and TT states through $V_{\mathrm{FE}-\mathrm{TT}}$, still it should be difficult to extract the coupling $V_{\mathrm{FE}-\mathrm{TT}}$ from Davydov splitting. Indeed, a recent theoretical study have shown negligible effects of the mixing with TT and FE states in the lowest lying absorption bands. ${ }^{64}$

We see that the energy stabilization in the FE state $\left(\Delta E_{\mathrm{FE}}\right.$ and $\left.J^{\text {eff }}\right)$ is primarily caused by the horizontal couplings $\left(F_{\mathrm{HH}}\right.$ and $F_{\mathrm{LL}}$ ) appearing in eqn (5) and (7), while that in the TT state $\left(\Delta E_{\mathrm{TT}}\right)$ by the non-horizontal couplings appearing in eqn (6). Therefore, if we could control each matrix element at will, we may address the desired energetic condition in a given chromophore. This issue is discussed in the rest part of this paper.

\subsection{Electronic coupling in covalently-linked systems}

In covalently-linked dimer systems investigated in this study, we evaluate the Fock matrix elements in eqn (1) by using the non-orthogonal effective Hamiltonian theory. ${ }^{52,65,66}$ This is again an effective Hamiltonian theory as described in eqn (2) but the Hamiltonian considered here is the Fock matrix. Since the detail of the theory is not needed for discussion, we here describe only the essential part, which is directly related to both the present analysis and our molecular design strategy. The detail of the theory is found in the ESI $\dagger$ in the previous paper. ${ }^{52}$ The correspondence of the following treatment of electronic couplings to that in many-electron wavefunction theory has been pointed out by Zeng et al. ${ }^{63}$

The orbital space spanned by atomic orbitals is divided into the subspace of interest called the model space $P$, which consists of the HOMOs and LUMOs of the chromophores, and the remaining subspace, called complement space $Q$, which consists of the rest of the MOs in the chromophores and in the bridges. The effective Fock operator as a function of the energy parameter (Fermi energy) $E$ is written as

$$
\hat{P}^{\dagger} \hat{F}^{\mathrm{eff}} \hat{P}=\hat{P}^{\dagger}(\hat{F}-E \hat{I}) \hat{P}+\hat{P}^{\dagger}(\hat{F}-E \hat{I}) \hat{G}_{\hat{Q}^{\dagger} \hat{Q}}(\hat{F}-E \hat{I}) \hat{P},
$$

where $G_{Q^{\dagger} Q}$ is the Green's function that describes the effect of the complement space $Q$ on the model space $P$. This formula approximately separates the total Fock matrix elements into the direct-overlap contribution in the first term and the bridgemediated contribution in the second term. These may be called through-space and through-bond couplings, respectively. The energy parameter $E$ is determined by iteratively solving eqn 
(9) so as to make $E$ match the average of eigenvalues obtained from eqn (9). The orbital basis spanning the subspaces are obtained through the diagonalization of the Fock matrix, ${ }^{52}$ which is evaluated from the conventional self-consistent-field calculation, in each fragment atomic orbital subspace, that is, the chromophore 1 and 2, and bridges $1,2, \ldots N$, and so on. Hence, the complement space $Q$ may be further decomposed into as,

$$
\hat{Q}=\hat{Q}^{\dagger} \hat{C}_{1} \hat{Q}+\hat{Q}^{\dagger} \hat{C}_{2} \hat{Q}+\hat{B}_{1}+\hat{B}_{2}+\ldots+\hat{B}_{N}
$$

where $C_{1}$ and $C_{2}$ indicate the orbital subspaces of the chromophores 1 and 2, respectively; $B_{1}, B_{2}, \ldots, B_{N}$ indicate the orbital subspaces of the bridges $1,2, \ldots, N$, respectively. The partitioning of the orbital space is schematically shown in Fig. 2.

The quantum interference in a bridge-mediated coupling may be understood both by the atomic orbital picture and by MO picture. For the former, for example, see reviews. ${ }^{67-69} \mathrm{We}$ here consider the MO picture, which is a hybrid view based on the use of localized MOs in each fragment (chromophores and bridges) and of delocalized MOs within a fragment. ${ }^{70}$ This picture is useful for describing the $\pi$-conjugated systems. We describe this at Hückel level of approximation as an introduction. ${ }^{52,70}$ For simplicity, we consider a singly-bridged chromophore dimer without direct-overlap, that is, two chromophores are spatially separated. In this level of approximation, the bridge-mediated coupling is expressed as ${ }^{52,70}$

$$
F_{i j}^{\text {eff bridge-mediated }} \approx c_{\mu^{\prime} i} c_{v^{\prime} j} \beta^{2}\left(\frac{c_{\mu \mathrm{HB}} c_{v \mathrm{HB}}}{E-E_{\mathrm{HB}}}-\frac{c_{\mu \mathrm{LB}} c_{v \mathrm{LB}}}{E_{\mathrm{LB}}-E}\right)
$$

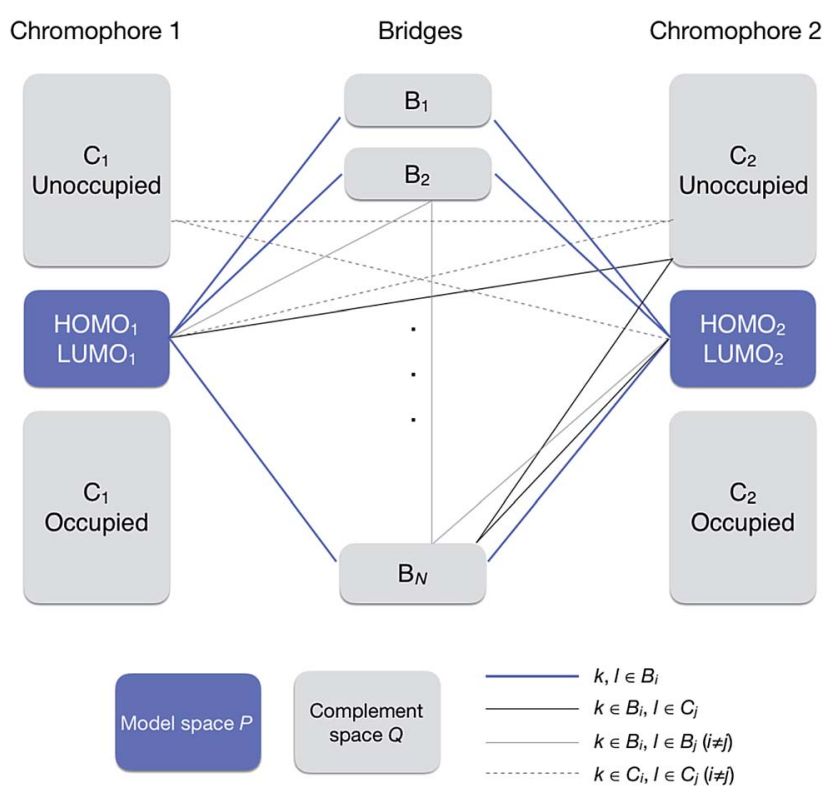

Fig. 2 Orbital subspaces and interaction paths (see eqn (10) and (13)). Solid blue line represents the interaction paths from a MO to another $\mathrm{MO}$ in the model space through a bridge, solid thick grey line through a bridge and a chromophore, solid thin grey line through two bridges, and broken grey lines through two chromophores. All the grey line terms are included in terms named "others" in eqn (12). where $\beta$ is the resonance integral; $c$ is a MO coefficient; subscripts $i$ and $j$ denote the HOMO or LUMO of the chromophores; HB and LB indicate the HOMO and LUMO of the bridge with orbital energies $E_{\mathrm{HB}}$ and $E_{\mathrm{LB}}$, respectively; Greek subscripts with and without prime indicate a pair of carbon atoms, where a fragment pair, that is, a chromophore and the bridge, is linked with the chemical bond $\mu^{\prime}-\mu$ or $\nu^{\prime}-\nu ; E$ denotes the Fermi energy level. As long as a bridge is bonded with chromophores having moderate MO amplitudes on the bonded sites, the prefactor of the parenthesis in eqn (11) should be moderate. In the parenthesis, we expect constructive or destructive quantum interference depending on the relative phase of the HOMO and LUMO in the bridge. Note that the denominators are usually positive. When only one side of the bridge sites, say $\mu$, changes in its MO sign between the HOMO and LUMO, the constructive quantum interference results in a large electronic coupling, while when none of the bridge sites changes, the destructive quantum interference results in a small electronic coupling. Therefore, we can control the amplitude of the electronic couplings $F_{i j}$ through the bridge design. A good example of constructive bridge is observed in an alternant-hydrocarbon bridge linked with chromophores at starred and non-starred carbon atoms, respectively, as shown in the previous study. ${ }^{52}$ In the above discussion, we have not focused on the sign of the electronic couplings but only on the amplitudes. On the other hand, relative signs of bridge-mediated couplings will be shown to play a crucial role in the following discussion for doubly or multiply-bridged systems.

We here discuss the sign of the electronic couplings and how they are related to molecular structures. Let us consider a linear bridge with two $\pi$-orbitals like ethylene, a kind of alternant hydrocarbons. The top view of the HOMO and LUMO of the model bridge is schematically shown in Fig. 3a. Two chromophores are linked at the left and right sides of the bridge, respectively. This case should correspond to the constructive quantum interference as explained above. We further assume

(a)

(b)

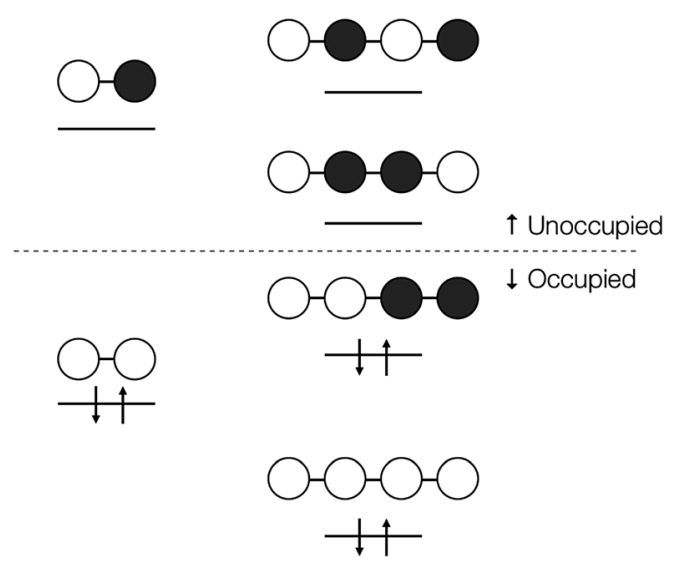

Fig. 3 Top view of orbital diagram for linear two $\pi$-electron system such as ethylene (a), and for linear four $\pi$-electron system such as butadiene (b). The signs of the HOMO at the edges in the former have the same sign, while those in the latter have mutually the opposite sign. 
that the signs of the MOs of the chromophores are positive at the linked sites, which is always possible without losing the generality when we consider a singly-bridged system. Then, the bridge-mediated coupling should be positive because the prefactor of the parenthesis in eqn (11) is positive and the term in the parenthesis is also positive. Next, we consider another bridge with four $\pi$-orbitals in a line like butadiene. The Frontier MOs of such kind of compounds are shown in Fig. 3b. Again, two chromophores are linked with the bridge at the ends of the bridge, where constructive interference is also expected. Assuming the same situation as that of ethylene bridge in the relative phase of chromophores, we should obtain a negative coupling $F_{i j}$, which is opposite to that obtained in the previous case, because the parenthesis term becomes negative. Consequently, we can predict the sign of the parenthesis term from the nature of $\pi$-orbitals in a bridge. The phase of bridgemediated coupling is uniquely defined through the relative phase of the HOMO and LUMO of a bridge at the positions linked to the chromophores, which is an intrinsic property of a bridge. Thus, these two kinds of intrinsically constructive patterns realized in these bridges may be referred to as "positively-constructive (PC)" and "negatively-constructive (NC)", respectively. When the two chromophore MOs have mutually the same signs at the linked sites, a PC bridge induces a positive coupling, while a NC bridge induces a negative coupling. On the other hand, when the two chromophore MOs have mutually the opposite signs at the linked sites, a PC bridge induces a negative coupling, while a NC bridge induces a positive coupling. Note that "positively-constructive" does not mean positive electronic coupling but does a positive value for the parenthesis term in eqn (11).

\subsection{Doubly-bridged systems and quantum interference between bridges}

In this section, we discuss the effect of quantum interference between $N$ bridges, where the relative signs of the electronic couplings are quite important. By partitioning the complement space $Q$ into subspaces as shown in eqn (10), the bridgemediated contribution may be partitioned into the sum of the contributions from each subspace, that is, from each bridge (see also Fig. 2):

$$
\begin{aligned}
&\left\langle i\left|\hat{P}^{\dagger} \hat{F}^{\text {eff }} \hat{P}\right| j\right\rangle^{\text {bridge-mediated }}= \sum_{k, l \in Q}\langle i|(\hat{F}-E \hat{I})| k\rangle\left\langle\tilde{k}\left|\hat{G}_{\hat{Q}^{\dagger} \hat{Q}}\right| l\right\rangle \\
& \times\langle\tilde{l}|(\hat{F}-E \hat{I})| j\rangle \\
& \sum_{k, l \in Q}=\sum_{k, l \in B_{1}}+\sum_{k, l \in B_{2}}+\ldots+\sum_{k, l \in B_{N}}+\sum_{k \in B_{1}, l \in B_{2}}+\ldots+\sum_{k, l \in\left(C_{1} \in Q\right)}+\ldots \\
&=\sum_{k, l \in B_{1}}+\sum_{k, l \in B_{2}}+\ldots+\sum_{k, l \in B_{N}}+\sum_{k, l \in Q}^{\text {others }}
\end{aligned}
$$

here, the first $N$ terms represent bridge-mediated contributions through one of the bridges $1,2, \ldots$, and $N$, respectively, which are indicated by solid blue lines in Fig. 2, while the rest includes those through a bridge and a chromophore, through two bridges, and through two chromophores, which are indicated by solid thick, solid thin and broken grey lines, respectively, in Fig. 2. A character with a tilde indicates a reciprocal basis that is orthogonal to the corresponding basis without a tilde. Again assuming that the contributions from the HOMO and LUMO of the bridges are dominant as done in eqn (13), we obtain

$$
\begin{aligned}
F_{i j}^{\mathrm{eff}} \text { bridge-mediated } \approx & \sum_{b}^{\text {all }} c_{\mu(b)^{\prime} i} c_{v(b)^{\prime} j} \beta^{2} \\
& \times\left(\frac{c_{\mu(b) \mathrm{HB}} c_{v(b) \mathrm{HB}}}{E-E_{\mathrm{HB}(b)}}-\frac{c_{\mu(b) \mathrm{LB}} c_{v(b) \mathrm{LB}}}{E_{\mathrm{LB}(b)}-E}\right)
\end{aligned}
$$

where a Greek character with the bridge index $(b)$ indicates the atom linked with the bridge $b(b=1,2, \ldots, N)$. Hence the total bridge-mediated coupling depends on the relative phase of linked sites of the Frontier MOs in chromophores as well as on the nature of the bridges (PC or NC). As in the case for singlybridged systems, the sign of the parenthesis is an intrinsic property of the bridge. The sign of the prefactor, however, depends on the linked position because we can no longer define the phase of the HOMO and LUMO of the chromophores as positive for all the linked sites $\mu(b)$ and $\nu(b)$ in any $b$. Therefore, the linked position is crucial for the total coupling in eqn (14). Note that eqn (14) is an approximate description of bridges, where we assume that bridges do not interact with each other. If this is not valid, the contributions referred to as "others" in eqn (12) may not be negligible.

Here, we consider how the linked position affects the couplings, and then classifies all the situations, which are generated by considering the combination of the MO phases and bridge choices, into the four essential cases. We here consider only the symmetric doubly-bridged dimer $(N=2)$. All the signs of the eight chromophore MO coefficients appearing in the prefactor in eqn (14) for two bridges, $c_{\mu(1)^{\prime} \mathrm{H} 1}, c_{\nu(1)^{\prime} \mathrm{H} 2}, c_{\mu(2)^{\prime}}$ ${ }_{\mathrm{H} 1}, c_{\nu(2)^{\prime} \mathrm{H} 2}, c_{\mu(1)^{\prime} \mathrm{L} 1}, c_{\nu(1)^{\prime} \mathrm{L} 2}, c_{\mu(2)^{\prime} \mathrm{L} 1}$ and $c_{\nu(2)^{\prime} \mathrm{L} 2}$, are related to the nature of the linked sites in the chromophores indicated, and the total patterns of them are $2^{8}=256$ when we consider plus or minus for these coefficients. They, however, can be reduced into two situations when we consider only symmetric dimers. This is essentially sufficient to classify the effect of the bridges in electronic couplings studied here. The classification is based on the signs of the products of the MO coefficients in the chromophores at the linked sites. The first situation of the simplest ones is (I) that the products have the same signs in all the relevant combinations (Table 1). The second situation is somewhat complicated and is (II) that the products have the same signs in all the relevant combinations except for the two products, that is, the product of the HOMO of the chromophore 1 and the LUMO of the chromophore 2, and also the product of the LUMO of the chromophore 1 and the HOMO of the chromophore 2, at the linked sites of only one of the bridges (Table $2)$. In the ESI, $\uparrow$ we show some examples of seemingly different but physically equivalent situations with that shown in Table 1. In addition to these essential two MO phase patterns in the chromophores at the linked sites, four ways of the choices in the bridge character are possible: PC-PC, NC-NC, PC-NC, and NC$\mathrm{PC}$, which indicate the character of bridge 1-2, respectively. 
Table 1 Products (prefactor in eqn (14)) of MO coefficient of chromophores in the case ()$^{a, b}$

\begin{tabular}{|c|c|c|c|c|}
\hline & H2@b1 & H2@b2 & L2@b1 & L2@b2 \\
\hline H1@b1 & + & & + & \\
\hline H1@b2 & & + & & + \\
\hline L1@b1 & + & & + & \\
\hline L1@b2 & & + & & + \\
\hline \multicolumn{5}{|c|}{$\begin{array}{l}{ }^{a} \mathrm{H} i @ \mathrm{~b} j(\mathrm{~L} i @ \mathrm{~b} j \text { ) represents an index of the MO coefficient of the HOMO } \\
(\mathrm{LUMO}) \text { of the chromophore } i \text { at the linked site with the bridge } j, c_{\mu(j)^{\prime} \mathrm{H}} \\
\left(c_{\mu(j)^{\prime} \mathrm{L} i}\right){ }^{b}+\text { indicates the positive sign of the product of MO coefficients } \\
\text { of the chromophore } 1 \text { shown in the left column and of } 2 \text { shown in the } \\
\text { top row. Blank cells represent that any signs of the product are } \\
\text { possible for this case. }\end{array}$} \\
\hline
\end{tabular}

Table 2 Products (prefactor in eqn (14)) of MO coefficient of chromophores in the case (II) $)^{a, b}$

\begin{tabular}{|c|c|c|c|c|}
\hline & H2@b1 & H2@b2 & L2@b1 & L2@b2 \\
\hline H1@b1 & + & & + & \\
\hline H1@b2 & & + & & - \\
\hline L1@b1 & + & & + & \\
\hline L1@b2 & & - & & + \\
\hline
\end{tabular}

${ }^{a} \mathrm{H} i @ \mathrm{bj}$ (Li@bj) represents an index of the MO coefficient of the HOMO (LUMO) of the chromophore $i$ at the linked site with the bridge $j, c_{\mu(j)^{\prime} \mathrm{H} i}$ $\left(c_{\mu(j)^{\prime} \mathrm{L} i}\right) \cdot{ }^{b}+$ and - indicate the positive and negative signs of a product of the MO coefficients of the chromophore 1 shown in the left column and of 2 shown in the top row, respectively. Blank cells represent that any signs of the product are possible for this case.

However, these four choices in the bridge character again can also be reduced into two, because the others give the essentially same electronic couplings except for their sign, which does not change the underlying physics. For example, the bridge choice of PC-PC gives the same electronic coupling matrix elements in their amplitudes with the opposite signs to those from the corresponding bridge choice NC-NC, given the MO amplitudes in the bridge moieties are the same. Thus, we do not distinguish them in this paper. In total, we have four cases in symmetric doubly-bridged dimers: (I-PP) the chromophore MO phases at the linked sites are in the pattern (I) and the combination of the bridges is PC-PC; (I-PN) the chromophore MOs are in the pattern (I) and the combination of the bridges is PC-NC; (II-PP) the chromophore MOs are in the pattern (II) and the combination of the bridges is PC-PC; (II-PN) the chromophore MOs are in the pattern (II) and the combination of the bridges is PCNC. As noted above, we refer to the case that is called (I-NN) as (I-PP).

The above essential four cases, that is, (I-PP), (I-PN), (II-PP) and (II-PN), are schematically shown in Fig. 4. Other cases that are not shown in Fig. 4 are physically equivalent to one of them, or are non-symmetric dimers, or are other linking patterns (see below). In Fig. 4, the phases of the HOMO and LUMO at the linked sites are expressed by white (positive) and black (negative) circles. A PC and NC are denoted by solid and broken lines, respectively. In the case (I-PP), in all the Fock matrix elements $F_{i j}$, the quantum interference between bridges

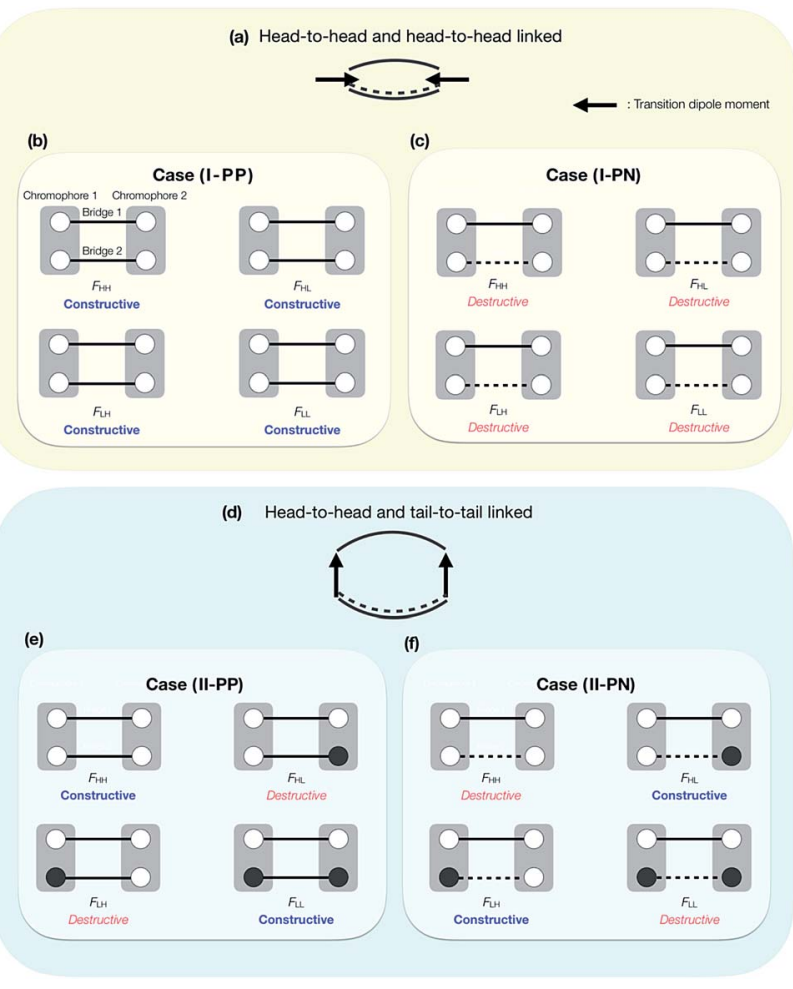

Fig. 4 Schematic picture of bridge linked patterns with head-to-head and head-to-head positions (concerning the transition dipole moments of chromophores) (a), and with heat-to-head and tail-to-tail positions (d), and of quantum interference between bridges in the cases (I-PP), (I-PN), (II-PP) and (II-PN) ((b), (c), (e) and (f)). Two chromophores (grey rectangular part) are linked by two bridges (black solid and/or broken lines). The phases of HOMO and LUMO at the linked sites are indicated by white (positive) and black (negative) circles. A positively-constructive and negatively-constructive bridges are described as black solid and broken lines, respectively.

is constructive, leading to large electronic coupling amplitudes in all the elements. In the case (I-PN), the situation is opposite: in all the Fock matrix elements $F_{i j}$, the quantum interference between bridges is destructive, leading to small electronic coupling amplitudes in all the elements. In the case (II-PP), due to the PC nature of the bridges 1 and 2 and mutually anti-phase HOMO-LUMO relation of chromophores at the two linked sites, constructive interference in horizontal couplings $\left(F_{\mathrm{HH}}\right.$ and $\left.F_{\mathrm{LL}}\right)$ results in large amplitudes of them, while destructive interference in non-horizontal couplings $\left(F_{\mathrm{HL}}\right.$ and $\left.F_{\mathrm{LH}}\right)$ results in small amplitudes of them. In the case (II-PN), the situation is opposite to the previous case: destructive interference in horizontal couplings results in small amplitudes of them, while constructive interference in non-horizontal couplings results in large amplitudes of them. In short, it is found that in the case (I$\mathrm{PP}$ ) (in the case (I-PN)), all the Fock matrix elements are large (small) in their amplitudes; in the case (II-PP) (in the case (II$\mathrm{PN})$ ), the horizontal couplings are large (small) in their amplitudes, while the non-horizontal couplings are small (large) in their amplitudes.

We give some comments on the characteristics and the potential for materials design using these four cases. The case 
(I-PP) seems to be a simple extension of singly-bridged systems, where the effect of a bridge is doubled when the same bridges are used. The difference between the singly-bridged systems and I-(PP) will be shown in ab initio calculations in this paper. The case (I-PN) may be a good way to design weak coupling system, which could lead to slow but high-yield SF and/or TTA systems. ${ }^{47,52,56,63}$ The lowest lying state can keep its electronic character from strong mixing with other states due to small electronic couplings from the view point of electronic configuration. At the same time, small electronic couplings, however, would suppress the transition rate between the FE and TT states, and could lead to deactivation pathways for the system before undergoing SF or TTA. This is an issue of dynamics, so that quantum dynamics simulation or rate estimation will answer the question of whether a system prefers. We do not discuss in detail this issue because we focus mainly on qualitative difference in electronic couplings and resulting energetics, and this is beyond the scope of the present paper. The cases (II-PP) and (II-PN) may be interesting because, from eqn (5)-(7), the relative energy balance between singlet and tripletpair excited states eqn (8) may be controlled by tuning the relative amplitude of the horizontal and non-horizontal couplings. These four cases are expected to correspond to the energy diagrams shown in Fig. 1a-d, respectively.

As noted above, Fig. 4 does not cover all the patterns of MOs in symmetric doubly-bridged systems, though other patterns are physically equivalent to one of the above four cases. In fact, the case (I-PP), which results in constructive quantum interferences in all the Fock matrix elements, is also found for other phase patterns of MOs, see Fig. S1. $\dagger$ This complexity may be avoided by considering the direction of the transition dipole moment of the HOMO to LUMO transition in a chromophore, which provides information of the relative phase of the MO, instead of the phases of MOs themselves. Schematic pictures of the relative direction of the transition dipole moments in the chromophores are shown in Fig. 4a and d, the former of which correspond to the cases (I-PP) and (I-PN), and the latter of which correspond to the cases (II-PP) and (II-PN), respectively. In this viewpoint, the cases (I-PP) and (I-PN) correspond to linking the chromophores at two head-to-head positions concerning the transition dipole moments of the chromophores, respectively, while the case (II-PP) and (II-PN) correspond to bridging at head-to-head and tail-to-tail positions. More precisely, local distribution of the transition density is found to classify the effects of the linked position into the cases (I-PP) and (I-PN), and into (II-PP) and (II-PN). This is approximately identified by taking a product of the HOMO and LUMO of the chromophore, which turns out to be identical to considering the relative phase of the MOs at the linked positions shown in Fig. 4. Other cases rather than these four cases, for example, asymmetrically bridged, triply- and multiply-bridged systems, head-to-tail bridged systems, and hetero dimer ${ }^{53,55}$ systems are possible and interesting, though we do not go further in this study. Electronic couplings in such systems can also be predicted in the same manner.

\section{Model systems}

We choose tetracene as the model chromophore in this study (Scheme 1a). Tetracene is known to have a slightly lower singlet excitation energy $E\left(\mathrm{~S}_{1}\right)=2.32 \mathrm{eV}$ than twice the triplet excitation energy $2 E\left(\mathrm{~T}_{1}\right)=2.50 \mathrm{eV}$ in crystal, ${ }^{71}$ while in gas phase, the singlet excitation energy is estimated to be $2.75-2.76 \mathrm{eV}^{41,72}$ (see Section 4), which is $0.25-0.26 \mathrm{eV}$ higher than twice the triplet excitation energy. The quantum yield of the fluorescence of unsubstituted tetracene in solution is reported as $14-16 \%,{ }^{73}$ and it reaches up almost unity by changing substituents. ${ }^{74}$ On the contrary, in solid phase, tetracene and its derivatives are known to induce high-yield $\mathrm{SF}^{\mathbf{1 3 - 1 9}}$ with providing essentially no fluorescence $\left(2 \times 10^{-5} \%\right.$ quantum yield $\left.{ }^{75}\right)$. This approximate isothermic condition of tetracene for SF and TTA motivates us to investigate the possibility of selective control of the Fock matrix elements suitable for SF or TTA on the basis of the perturbation analysis in Section 2.1. The product of the HOMO and LUMO, which represents the approximate transition density, is found to be positive on the one of the zigzag edge, while negative on the other zigzag edge (see the ESI $\dagger$ ). Hence, we expect that the cases (I-PP) and (I-PN) could be realized when two tetracenes are linked by two bridges at the same side of the zigzag edge carbon atoms, and that the cases (II-PP) and (II-PN) could be realized when linked at the armchair edge carbon atoms. We consider polyyne with $n$ ethynyl units as a bridge. Polyynes can be utilized for linking covalently many kinds of chromophores with different ethynyl unit lengths. ${ }^{76-82}$ As in the cases of polyene bridges discussed in Section 2.2, a polyyne also should act as a PC bridge when odd $n=1,3,5, \ldots$, and a NC bridge when even $n=2,4,6, \ldots$. By using two polyynes with the lengths $m$ and $n$, we consider two model dimers 1-(m, $\boldsymbol{n})$, where

(a)

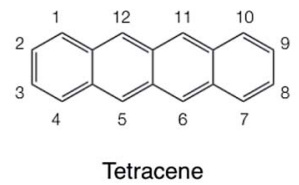

(b)
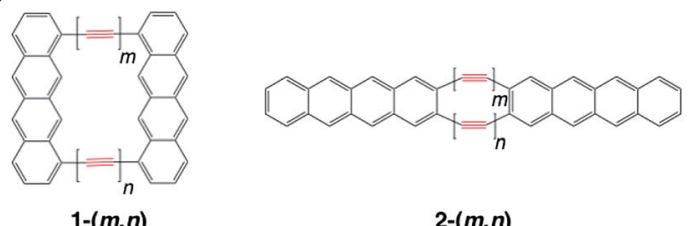

$1-(m, n)$

$2-(m, n)$

(c)

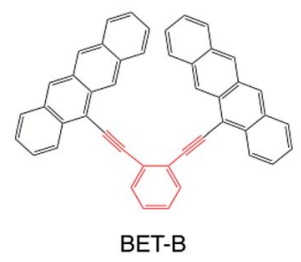

Scheme 1 Molecular structure tetracene (a), model tetracene dimers (b) and experimentally investigated tetracene dimer (c). ${ }^{49}$ Here, $(m, n)=$ $(1,1),(2,1),(2,2),(3,1),(3,2)$ and $(3,3)$. BET-B = ortho-bis(5-ethynyltetracenyl) benzene. Bridge moieties are indicated by red lines. 
the chromophores are linked through the polyynes at $\mathrm{C}_{1}$ and $\mathrm{C}_{10}$ atoms, and 2-( $\boldsymbol{m}, \boldsymbol{n})$, where the chromophores are linked through the polyynes at $\mathrm{C}_{2}$ and $\mathrm{C}_{3}$ atoms (see Scheme $1 \mathrm{~b}$ ). For comparing the performance of the model dimer with others reported in previous studies, we also consider ortho-bis(5ethynyltetracenyl)benzene (BET-B). This molecule was shown to undergo SF very efficiently with the triplet yield $1.54 \pm 0.10$ (1.54 triplet excitons per singlet exciton) with the time constant of $2 \mathrm{ps}$ in its thin film,$^{49}$ which is the highest triplet yield and the fastest SF in covalently-linked tetracene dimers reported ever. ${ }^{41,42,46,49}$

\section{Computational details}

Molecular geometries were optimized by using density functional theory with the Grimme's dispersion correction B97-D xcfunctional ${ }^{83}$ at their highest possible point group, except for 1$(\mathbf{1}, \mathbf{1})$, where the $D_{2 \mathrm{~h}}$ geometry was found to have an imaginary frequency and thus reduced to $C_{2 \mathrm{~h}}$. The long-range correction scheme $^{84}$ combined with the Becke-Lee-Yang-Parr exchangecorrelation functional ${ }^{84,85}$ (LC-BLYP) was used for the electronic coupling calculations, where the range-separating parameter $\mu$ is set to $0.33 \mathrm{bohr}^{-1}{ }^{86}$ The electronic couplings were evaluated by using eqn (9). The Frontier MOs in the chromophores were obtained through the diagonalization of the Fock matrix, which is evaluated in the whole molecule, in the subspace of each fragment, that is, $C_{1}, C_{2}, B_{1}$ and $B_{2}$, respectively, as was done in the previous study. ${ }^{52}$ In order to evaluate $J_{\text {Coul }}$, we considered hydrogen-capped chromophores. After the geometry optimization in the model molecules $\mathbf{1 -}(\boldsymbol{m}, \boldsymbol{n})$ and $\mathbf{2}-(\boldsymbol{m}, \boldsymbol{n})$, the bridge moiety (polyyne part) and the other chromophore are removed, and two hydrogen atoms are attached onto the remaining tetracene moiety instead of the removed parts. The position of attached hydrogen atoms were optimized under the restriction of the other atoms being fixed. After this, $J_{\text {Coul }}$ was computed from the Coulomb interaction between the Mülliken transition atomic charges obtained from the time-dependent density functional theory calculation using LC-BLYP for each isolated tetracene, as was done in the previous study. ${ }^{58}$ Pople's 6-31G** basis $\operatorname{set}^{87}$ was used for all the calculations. All the quantum chemistry calculations were performed by using Gaussian 09 program package..$^{88}$ The effective Hamiltonian matrix elements were evaluated by using our house code.

The relative energy of the FE and TT states is crucial for determining whether SF or TTA tends to occur. Here, we consider the $0-0$ absorption band energy for state $\mathrm{X}$ relative to the ground state as

$$
E_{\mathrm{X}}=E_{\mathrm{X}}^{\mathrm{FC}}+E_{\mathrm{X}}^{\mathrm{vib}}+E_{\mathrm{X}}^{\mathrm{pol}}+E_{\mathrm{X}}^{\text {mix }}
$$

where $E_{\mathrm{X}}^{\mathrm{FC}}$ is the Frank-Condon energy, $E_{\mathrm{X}}^{\mathrm{vib}}$ is the reorganization energy and the zero-point energy correction, $E_{\mathrm{X}}^{\mathrm{pol}}$ is the polarization effect through environment response upon the excitation, $E_{\mathrm{X}}^{\text {mix }}$ is the configuration interaction effect such as the FE-FE and FE-CT states mixing. Although the vibrational motion could play an important role in dynamical change in the relative energy, ${ }^{20,22,89}$ electronic coupling ${ }^{45,90}$ and dynamics, ${ }^{91-94}$ we here assume that it gives merely an energetic contribution to the excitation energy in a chromophore, $E_{\mathrm{X}}^{\mathrm{v} \text { ib }}$. As we consider the electronic Hamiltonian eqn (1), we have to estimate the singlet and triplet state energy without $E_{\mathrm{X}}^{\text {mix }}$ contribution in order to avoid double counting of the effect. As the triplet state is considered to be insensitive to the configuration interaction between neighbouring triplet excitons, which corresponds to narrow band width and spatial localization of a triplet exciton as is well known, the solid state absorption band $E(T)=1.25 \mathrm{eV}^{71}$ is expected to be regarded as an approximation to $E(\mathrm{~T})$ without $E_{\mathrm{X}}^{\text {mix }}$. Hence, we set $E_{\mathrm{TT}}=2 E(\mathrm{~T})=2.50 \mathrm{eV}$ in eqn (1).

The singlet excited state $0-0$ absorption band excluding the polarization effect, that is, the excitation energy in gas phase, can be estimated as $E_{\mathrm{S} 1}$ (gas) $=2.75-2.76 \mathrm{eV}^{41,72}$ by combining the solution spectra and empirical correction for solvation effect $\left(E_{\mathrm{S} 1}(\right.$ gas $)=E_{\mathrm{S} 1}($ solution $\left.)+\Delta^{\text {sol }}\right) \cdot{ }^{95}$ Typical values of this correction, which may be regarded as the polarization effect in solution, for the HOMO-LUMO singly-excited singlet state of polyaromaric hydrocarbons range $900-1500 \mathrm{~cm}^{-1}=0.11-$ $0.19 \mathrm{eV}$ depending on solvents. ${ }^{95}$ Here, we consider this correction for $E_{\mathrm{S} 1}$ (gas) in order to obtain a balanced treatment both for singlet and triplet exciton, where the latter $E_{\mathrm{T} 1}=$ $1.25 \mathrm{eV}$ includes the polarization effect in condensed phase. We set the correction as $0.15 \mathrm{eV}$, and hence, $E_{\mathrm{FE}}$ (including polarization effect) $=2.76-0.15=2.61 \mathrm{eV}$, where the solvent effect corresponds to benzene solution. Consequently, we assume slightly higher energy of FE state than TT state, $E_{\mathrm{TT}}-E_{\mathrm{FE}}=$ $-0.11 \mathrm{eV}$. This means that SF is exothermic, while TTA is endothermic in the weak electronic coupling limit, that is, $E_{\mathrm{X}}^{\text {mix }}=0$.

The location of the CT state energy is still under debate. The electroabsorption spectra in tetracene crystal gave $E_{\mathrm{CT}}=2.71-$ $3.063 \mathrm{eV},{ }^{96}$ depending on the dimer-pairs in crystal. Using these values, theoretical modelling including FE and CT states with vibronic effect succeeded in reproducing the absorption spectra in tetracene crystal. ${ }^{97}$ Although it can be different for each dimer, we assume $E_{\mathrm{CT}}=E_{\mathrm{FE}}+0.3=2.91 \mathrm{eV}$, which is in above values, for all the dimers. The CT energy dependence will be discussed in Section 5.4, otherwise the CT energy is fixed at $2.91 \mathrm{eV}$.

\section{Results and discussion}

\subsection{Fock matrix element}

Table 3 represents the evaluated electronic couplings of the dimers 1- $(\boldsymbol{m}, \boldsymbol{n})$ and $\mathbf{2}-(\boldsymbol{m}, \boldsymbol{n})$. Obviously, in $\mathbf{1}-(\boldsymbol{m}, \boldsymbol{n})$, in the even combinations, that is, $m+n=$ even, all the Fock matrix elements are found to be large and to lie in the range of 118.3$334.6 \mathrm{meV}$ in their amplitudes except for $F_{\mathrm{LL}}$ in 1-(1, 1), while in the odd combinations, that is, $m+n=$ odd, they are found to be relatively small and to lie in the range of $13.9-63.7 \mathrm{meV}$. This is what we expected from our discussion in Section 2.3, and the even and odd combinations should correspond to the cases (IPP) and (I-PN), respectively. We note that the difference between $F_{\mathrm{HH}}$ and $F_{\mathrm{LL}}$, which is important for large $V_{\mathrm{FE}-\mathrm{TT}}$, is also large for the case I-(PP). This is explained by the doubling of the residue terms from the next-nearest-neighbour interaction. ${ }^{52}$ 
Table 3 Fock Matrix elements in meV evaluated at LC-BLYP/6-31G** level of theory combined with non-orthogonal effective Hamiltonian approach

\begin{tabular}{|c|c|c|c|c|}
\hline \multirow[b]{2}{*}{ Molecule } & \multicolumn{2}{|c|}{ Horizontal coupling } & \multicolumn{2}{|c|}{$\begin{array}{l}\text { Non-horizontal } \\
\text { coupling }\end{array}$} \\
\hline & $F_{\mathrm{HH}}$ & $F_{\mathrm{LL}}$ & $F_{\mathrm{HL}}$ & $F_{\mathrm{LH}}$ \\
\hline $1-(1,1)$ & 334.6 & -89.6 & 284.8 & 284.8 \\
\hline $1-(2,1)$ & 63.7 & 38.9 & 44.4 & 44.4 \\
\hline $1-(2,2)$ & -247.8 & -154.5 & -237.2 & -237.2 \\
\hline $1-(3,1)$ & 285.0 & 176.7 & 251.6 & 251.6 \\
\hline $1-(3,2)$ & -34.9 & -13.9 & -37.0 & -37.0 \\
\hline $1-(3,3)$ & 188.8 & 118.3 & 160.7 & 160.7 \\
\hline $2-(1,1)$ & 263.4 & 291.6 & 0.0 & 0.0 \\
\hline $2-(2,1)$ & 58.5 & 20.9 & -239.7 & -239.7 \\
\hline $2-(2,2)$ & -180.0 & -181.9 & 0.0 & 0.0 \\
\hline $2-(3,1)$ & 214.0 & 175.8 & -72.1 & -72.1 \\
\hline $2-(3,2)$ & -26.7 & -12.8 & 170.3 & 170.3 \\
\hline $2-(3,3)$ & 139.1 & 129.1 & 0.0 & 0.0 \\
\hline
\end{tabular}

By changing the linked pattern between chromophores and bridges from that of 1- $(\boldsymbol{m}, n), \mathbf{2}-(\boldsymbol{m}, n)$ shows large horizontal couplings of 129.1-291.6 meV and small non-horizontal couplings 0-72.1 meV in their amplitudes in the even combinations, while shows small horizontal couplings $12.8-58.5 \mathrm{meV}$ and large non-horizontal couplings $170.3-239.7 \mathrm{meV}$ in their amplitudes in the odd combinations. These even and odd combinations in 2-( $\boldsymbol{m}, \boldsymbol{n})$ should correspond to the cases (II-PP) and (II-PN), respectively.

Here we have clearly demonstrated that we can design horizontal and non-horizontal couplings separately through tuning the bridge character, $\mathrm{PC}-\mathrm{NC}$, and the linked position. In order to confirm the correspondence between the results shown in this section and the theory provided in Section 2.3, we perform a further analysis of these couplings in the next section.

\subsection{Decomposition analysis}

The Fock matrix elements, both horizontal and non-horizontal couplings, are decomposed into the direct-overlap contribution, each bridge-mediated contribution and other contributions by using eqn (10) and (12). Here only bridge $\pi$-orbitals are considered as the bridge orbital subspaces. The results are shown in Fig. 5 for $\mathbf{1}-(\boldsymbol{m}, \boldsymbol{n})$ and Fig. 6 for $\mathbf{2}-(\boldsymbol{m}, \boldsymbol{n})$. The results show clear relationship between the Fock matrix elements, even-odd combinations and linked positions. In all the $(m, n)$ combinations of $\mathbf{1}$ and $\mathbf{2}$, each bridge-mediated coupling within a bridge is shown to be constructively enhanced, resulting in their large amplitudes. However, the signs of a coupling for a bridge as well as for the other bridge depend on $(m, n)$ combinations and the linked positions of the fragments. This is the quantum interference between bridges. In 1- $(\boldsymbol{m}, \boldsymbol{n})$ with the even combination, all the Fock matrix elements are found to be enhanced through constructive quantum interference between the bridges (Fig. 5a, c, d and f), while in the odd combination of 1- $(\boldsymbol{m}, \boldsymbol{n})$, all the Fock matrix elements are found to be suppressed by destructive quantum interference (Fig. 5b and e).
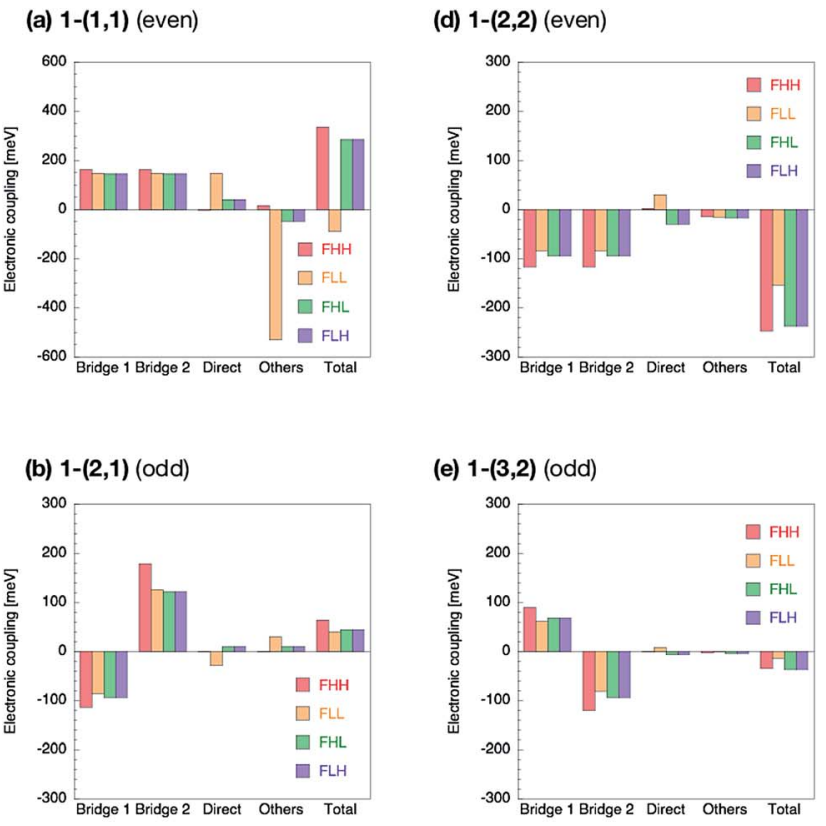

(c) 1-(3,1) (even)

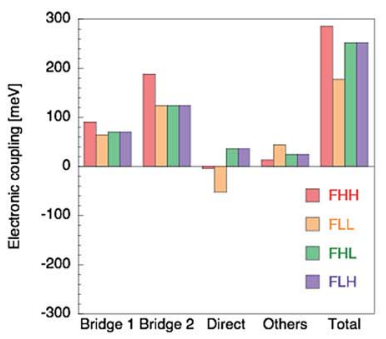

(f) 1-(3,3) (even)

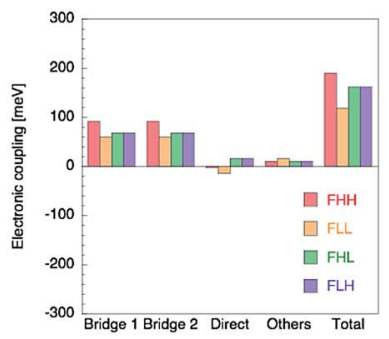

Fig. 5 Decomposition of the Fock matrix elements in 1- $(m, n)$ into the two bridge-mediated contributions from the bridges 1 and 2, directoverlap contribution, and others. Here, $(m, n)=(1,1)(a),(2,1)(b),(3,1)$ $(c),(2,2)(d),(3,2)(e)$, and $(3,3)(f)$. The longer bridge is referred to as bridge 1.

Therefore, these are considered to correspond to the cases (I-PP) and (I-PN), respectively. Note that $\mathbf{1 - ( 1 , 1 )}$ has non-negligible contribution from direct-overlap contribution and has contribution other than the bridges. This might be due to distortion of the $\pi$-plane in the chromophores, and also be related to the sensitivity to the localization method used to evaluate the couplings in the previous study. ${ }^{98}$ Enormous contributions in $F_{\text {LL }}$ other than direct and $\pi$-orbital bridge-mediated terms is partly due to non-negligible interactions with $\sigma$-orbitals of bridges (see ESI $\dagger$ ). To resolve this strange behaviour for such a kind of compounds, we might need further development of the present theory, which is left for the future investigation.

The situation is quite different in $\mathbf{2}-(\boldsymbol{m}, \boldsymbol{n})$. In the even combinations (Fig. 6a, c, d, and f), we find that the horizontal couplings have the same sign contributions from both the bridges, while the non-horizontal couplings have the mutually opposite sign contributions from each bridge. On the contrary, in the odd combinations (Fig. 6b and e) we find that the horizontal couplings have the mutually opposite sign contributions 
(a) 2-(1,1) (even)

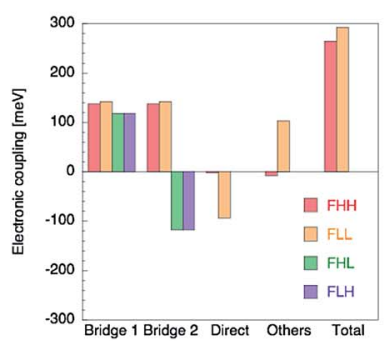

(b) 2-(2,1) (odd)

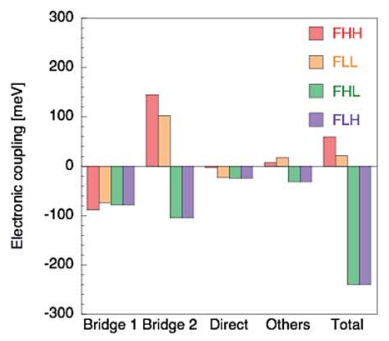

(c) 2-(3,1) (even)

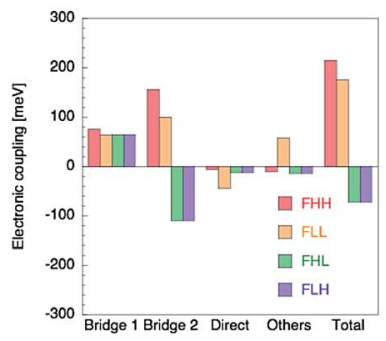

(d) 2-(2,2) (even)

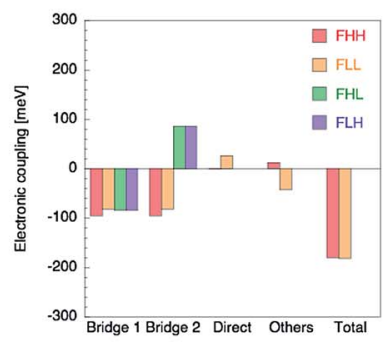

(e) 2-(3,2) (odd)

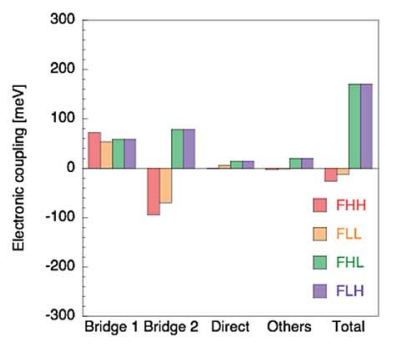

(f) 2-(3,3) (even)

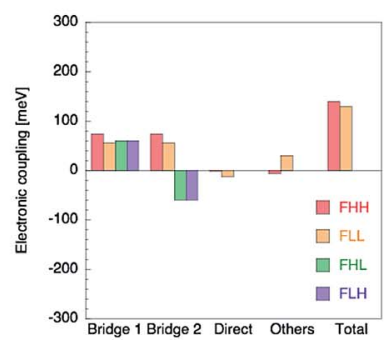

Fig. 6 Decomposition of the Fock matrix elements in $2-(m, n)$ into the two bridge-mediated contributions from the bridges 1 and 2, directoverlap contribution, and others. Here, $(m, n)=(1,1)(a),(2,1)(b),(3,1)$ $(c),(2,2)(d),(3,2)(e)$, and $(3,3)(f)$. The longer bridge is referred to as bridge 1.

from each bridge, while the non-horizontal couplings have the same sign contributions from both the bridges. As a result, it is found that the even combinations in $\mathbf{2}-(\boldsymbol{m}, \boldsymbol{n})$ give the large horizontal and small non-horizontal couplings, while that the odd combinations do the small horizontal and large nonhorizontal couplings. The even and odd combinations in 2-( $\boldsymbol{m}$, $n$ ) should correspond to the cases (II-PP) and (II-PN), respectively.

Zero non-horizontal couplings in $\mathbf{2}-(\boldsymbol{n}, \boldsymbol{n})(m=n)$, that is, the sum of the direct, bridge-mediated and other terms in them, may also be explained and understood by symmetry. Note that the point group of localized diabatic wavefunctions such as the HOMO and LUMO of chromophores in $2-(n, n)$ no longer belongs to the $D_{2 \mathrm{~h}}$ point group but to $C_{2 \mathrm{v}}$ point group, where the $C_{2}$ rotation axis lies along the long axis of the molecule. Therefore, the HOMO of a chromophore belongs to the $A_{2}$ irreducible representation and the LUMO of the other chromophore belongs to the $B_{1}$ irreducible representation in the $C_{2 \mathrm{v}}$ point group, respectively, so that the Fock matrix elements $F_{\mathrm{HL}}$ and $F_{\mathrm{LH}}$ vanish. A role of symmetry in covalently-linked SF systems was also discussed in several papers. ${ }^{\mathbf{1 , 2 2 3 , 4 5 , 9 2}}$ Unfortunately, although this explanation based on symmetry gives a clear insight into a part of electronic couplings in covalentlylinked systems, this is not sufficient for other compounds and for horizontal couplings investigated in the present study. For example, smaller non-horizontal coupling in $2-(3,1)$ than $2-(3$, 2) in spite of shorter bridge length of the former cannot be explained by symmetry nor in an intuitive way. The comprehensive understanding of the difference in the electronic couplings between these compounds $1-(m, n)$ and $2-(m, n)$ should be accomplished through the consideration of the quantum interference.

\subsection{Perturbation analysis}

In this section, we provide the perturbation analysis of model systems of the cases (I-PP), (I-PN), (II-PP) and (II-PN) in order to clarify the effect of electronic couplings on the energetics. As shown in eqn (5)-(8) as well as in Fig. 1, the relative energy of the FE and TT states after the CT-mixing through the horizontal and non-horizontal couplings strongly depends on the coupling strength and the CT state energy. Here, we have fixed the CT state energy as $2910 \mathrm{meV}$, which is $300 \mathrm{meV}$ higher than $\mathrm{FE}$ state. The evaluated second-order contributions to the FE and TT state energies as well as to the excitonic coupling are summarized in Table 4. The even-odd parity dependence on each energy correction, coupling and energetics are summarized in Table 5 .

First, we discuss the results of the perturbation analysis in 1$(\boldsymbol{m}, \boldsymbol{n})$ (see Table 4). The amplitude of the energy correction to the FE state through the CT-mixing, $\Delta E_{\mathrm{FE}}$, is found to be moderate in its amplitude for all the even combinations, while small for all the odd combinations. The order in the amplitude is, 1- $(1,1)\left(\Delta E_{\mathrm{FE}}=-566.9 \mathrm{meV}\right)>\mathbf{1}-(\mathbf{3}, \mathbf{1})(-374.9 \mathrm{meV})>\mathbf{1}-(\mathbf{2}, 2)$ $(-284.2 \mathrm{meV})>\mathbf{1}-(\mathbf{3}, 3)(-165.4 \mathrm{meV})>\mathbf{1}-(\mathbf{2}, \mathbf{1})(29.3 \mathrm{meV})>\mathbf{1}-(\mathbf{3}$, 2) $(-4.7 \mathrm{meV})$. It is found that longer bridges give less correction than short one $\mathbf{1 -}(\mathbf{1}, \mathbf{1})$ does when $m=n$. The energy correction to the FE state is found to be much more significant for even combinations than for odd combinations by one to two orders in its magnitude. The energy correction to the TT state, CTmediated excitonic coupling $J_{\mathrm{CT}}$ and FE-TT coupling $V_{\mathrm{FE}-\mathrm{TT}}$ are found to have the same even-parity dependence as that to the FE state. Hence, the electronic transition between the FE and TT states, that is, SF or TTA, is expected to be faster in the even combinations than odd combinations of $1-(m, n)$. The Coulomb contribution in the excitonic coupling, $J_{\text {Coul }}$, is found to be much smaller than $J_{\mathrm{CT}}$ in the even combinations. The effective energy difference defined by eqn (8) is shown to be positive for all the even combinations and negative for odd combinations, which indicates that the former molecules favour TTA rather than SF, while the latter favour SF rather than TTA.

Next, we discuss the results of the perturbation analysis in 2$(\boldsymbol{m}, \boldsymbol{n})$ (see Table 4). The amplitude of the energy correction to the FE state $\left|\Delta E_{\mathrm{FE}}\right|$ is found to be moderate in its amplitude for all the even combinations, while small for all the odd 
Table 4 CT-mediated energy perturbation to $\mathrm{FE}$ and $\mathrm{TT}$ states, Coulomb and CT-mediated excitonic coupling, and FE-TT coupling of tetracene dimers in meV evaluated at LC-BLYP/6-31G** level of theory $^{a}$

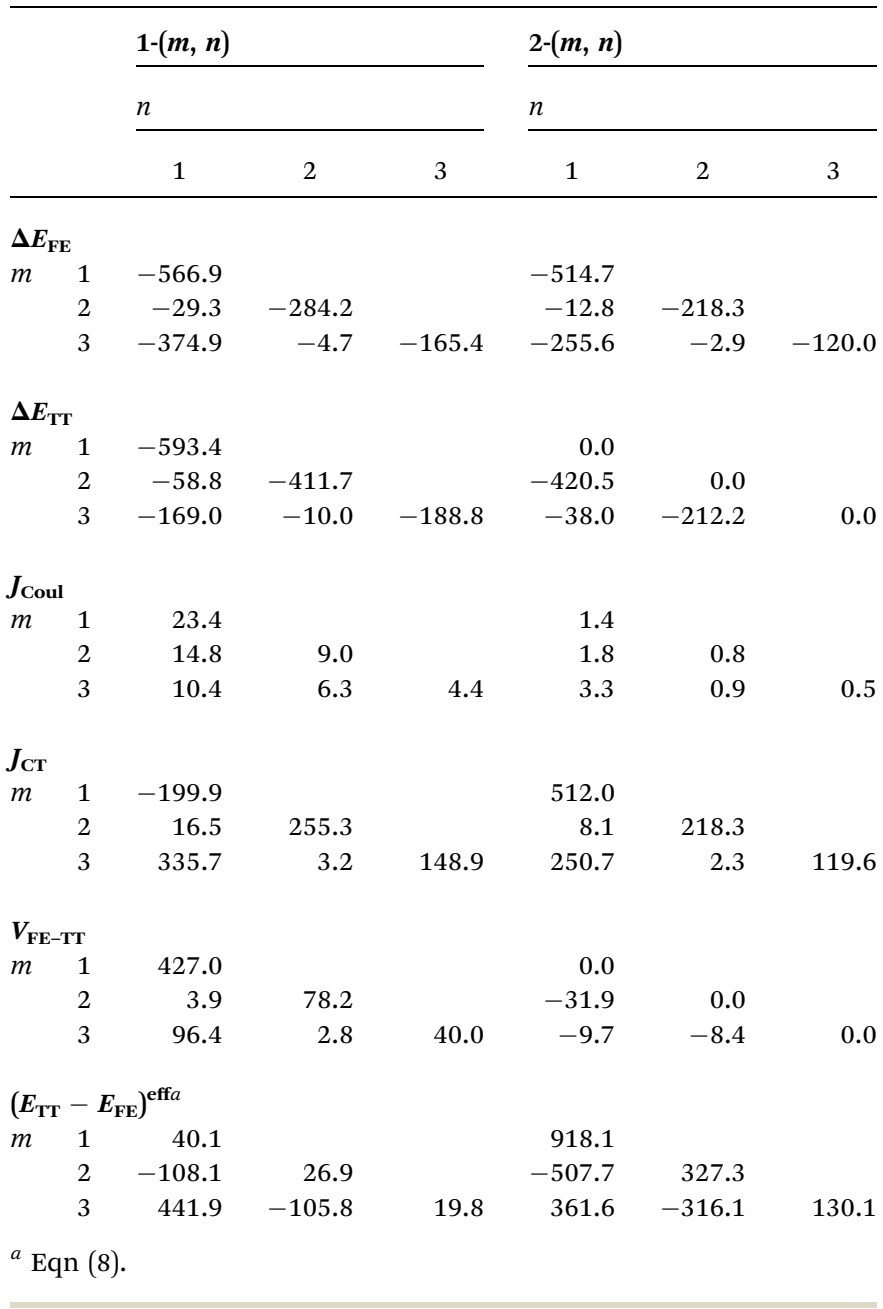

combinations. This seems to be the same as the situation found in 1- $(\boldsymbol{m}, \boldsymbol{n})$. The CT-mediated excitonic coupling $J_{\mathrm{CT}}$, which is found to be much larger than $J_{\text {Coul }}$, shows the same tendency as the energy correction to the FE state $\Delta E_{\mathrm{FE}}$. In contrast to $\Delta E_{\mathrm{FE}}$ and $J_{\mathrm{CT}}$, which are significant in the even combinations in both 1- $(\boldsymbol{m}, \boldsymbol{n})$ and 2-(m,n), the energy correction to the TT state $\Delta E_{\mathrm{TT}}$ in 2 - $(\boldsymbol{m}, \boldsymbol{n})$ shows the opposite tendency in the even-odd parity; the odd combinations have much larger $\left|\Delta E_{\mathrm{TT}}\right|$ than that of the even combinations. The FE-TT coupling is found to be the
Table 6 CT-mediated energy corrections, Coulomb coupling, and effective energy difference evaluated by LC-BLYP/6-31G** in meV

\begin{tabular}{llllll}
\hline$\Delta E_{\mathrm{FE}}$ & $\Delta E_{\mathrm{TT}}$ & $J_{\mathrm{Coul}}$ & $J_{\mathrm{CT}}$ & $V_{\mathrm{FE}-\mathrm{TT}}$ & $\left(E_{\mathrm{TT}}-E_{\mathrm{FE}}\right)^{\text {eff }}$ \\
\hline-232.0 & -1955.5 & 23.5 & 79.5 & 390.8 & -1730.6 \\
\hline
\end{tabular}

largest in the odd combination 2-(2, 1) $\left(\left|V_{\mathrm{FE}-\mathrm{TT}}\right|=39.1 \mathrm{meV}\right)$, the second largest in $2-(3,1)(9.7 \mathrm{meV})$ and then $2-(3,2)(8.4$ $\mathrm{meV})$. We see that the even combinations with $m=n$ have no $V_{\mathrm{FE}-\mathrm{TT}}$ due to zero non-horizontal couplings as shown above. We find that the effective energy difference in $2-(m, n)$ is positive in even combinations, while negative in odd combinations. This is considered as the result from the difference in the even-odd parity dependence between the horizontal-coupling-induced terms $\left(\left|\Delta E_{\mathrm{FE}}\right|\right.$ and $\left.\left|J_{\mathrm{CT}}\right|\right)$ and the non-horizontal-couplinginduced term $\left(\left|\Delta E_{\mathrm{TT}}\right|\right)$, where the former two terms are found to be large in the even combinations, while the latter term is found to be large in the odd combinations (see Table 5). From these considerations, we expect that the lowest excited state of the even combination molecules of $2-(\boldsymbol{m}, \boldsymbol{n})$ tends to become FE dominated and therefore favours TTA rather than SF, while that of the odd combination molecules of $2-(\boldsymbol{m}, \boldsymbol{n})$ tends to become TT dominated and therefore favours SF rather than TTA.

It is interesting to compare the present model molecules with previously reported efficient SF systems in order to clarify the applicability of the present approach. In contrast to most previous studies on covalently-linked tetracene dimers, ${ }^{41,42,46}$ which reported that the SF is endothermic and thereby inefficient, Korovina et al. reported that highly efficient SF in the covalently-linked tetracene dimer, BET-B, see Scheme $1 .^{49}$ The SF time constant and triplet yield were reported to be in the range of $0.8-2 \mathrm{ps}$ depending on the media and $154 \pm 10 \%$, respectively ${ }^{49}$ Its efficient SF was also confirmed by theoretical calculations, which indicate that the strong electronic coupling pushes the TT state below the FE state..$^{50}$ In order to compare our model molecules with this result, we evaluate the Fock matrix elements, energy correction and effective coupling matrix elements of BET-B (see Table 6). Clearly, we see the large non-horizontal couplings as compared to the horizontal ones, the formers of which mainly originate from the direct-overlap contribution (see the ESI $\dagger$ ), indicating a much stronger stabilization in the TT state than in the FE state. As expected, the effective energy difference is largely negative, $\left(E_{\mathrm{TT}}-E_{\mathrm{FE}}\right)^{\text {eff }}=$ $-1730.6 \mathrm{meV}$, which corresponds to energetically favourable SF.

Table 5 Parity dependence of cases, Fock matrix elements, CT-mediated energy corrections and coupling, and lowest state expected

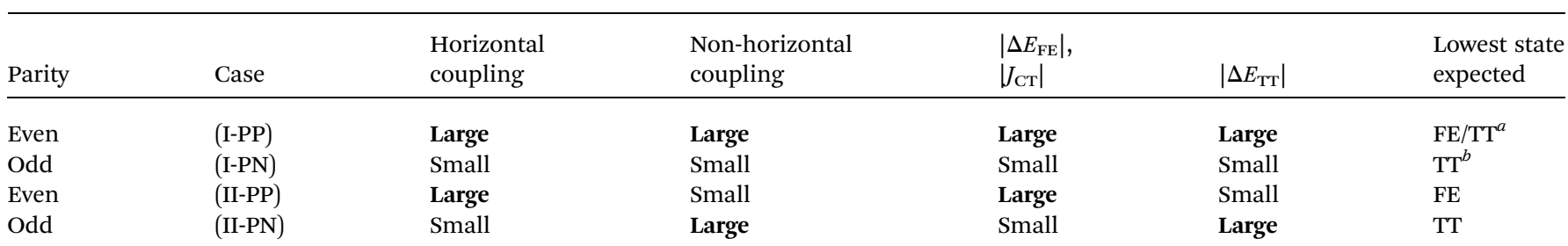

${ }^{a}$ CT energy sensitive. ${ }^{b}$ Assuming the condition $E_{\mathrm{TT}}<E_{\mathrm{FE}}$ at the monomer level. 
Besides, we also find the large $V_{\mathrm{FE}-\mathrm{TT}}=390.8 \mathrm{meV}$. Judging from these results, we conclude that highly efficient SF in BET-B is due to these large non-horizontal couplings as compared to the horizontal couplings, which favour the energetically exothermic condition, and due to the large $V_{\mathrm{FE}-\mathrm{TT}}$, which induces very fast SF. Of course, we cannot expect nearly $2 \mathrm{eV}$ stabilization through the electronic coupling in the TT state, which is clearly beyond the perturbation region. Despite that, we certainly obtained qualitative agreement with the experiment ${ }^{49}$ and the theoretical calculation ${ }^{50}$ with more sophisticated methodology. This example highly motivates us to further investigate the possibility of our designed molecules, especially the case (II-PN) $(2-(2,1)$ and $2-(3,2))$ for SF, where the non-horizontal couplings are much larger than the horizontal couplings. This is in the same situation as the case of BET-B, though here we have achieved that through the control of bridge-mediated contribution. Although the coupling, $V_{\mathrm{FE}-\mathrm{TT}}$, and exothermicity $\left|\left(E_{\mathrm{TT}}-E_{\mathrm{FE}}\right)^{\text {eff }}\right|$ are small as compared to those in BET-B, fast SF is expected for both of them $\left(\tau_{\mathrm{SF}}=120-300 \mathrm{ps}\right.$ in 2-(2, 1) and 1.7-4.3 ns in 2-(3, $2)$ ), by optimistically assuming that the SF rate is proportional to the square of $V_{\mathrm{FE}-\mathrm{TT}}$ with exothermic conditions $\left(\tau_{\mathrm{SF}} / \tau_{\mathrm{SF}}^{\mathrm{BET}-\mathrm{B}}=\right.$ $\left.\left|V_{\mathrm{FE}-\mathrm{TT}} / V_{\mathrm{FE}-\mathrm{TT}}^{\mathrm{BET}}\right|^{2}\right)$. These are still two or three orders faster than the previously reported low yield tetracene dimers. As previously reported non-radiative and radiative decay time constants for most tetracene dimers are in the order of ten nanoseconds, ${ }^{42,46,49} 2-(2,1)$ and $2-(3,2)$ should be promising candidates for SF, though a faster non-radiative decay in 500 ps was reported for BET-B.

We note an interesting point in the effective energy difference eqn (8) for covalently-linked systems. Using rough but reasonable approximations (see the ESI $\dagger$ ), we can show that the effective energy difference is always higher than the energy difference without the CT-mixing,

$$
\left(E_{\mathrm{TT}}-E_{\mathrm{FE}}\right)^{\mathrm{eff}}>E_{\mathrm{TT}}-E_{\mathrm{FE}}(=-0.11 \mathrm{eV})
$$

This is derived for covalently-linked dimers with a constructive bridge, two constructive bridges of the cases (I-PP) and (IIPP), where these cases give large amplitudes in all or horizontal part of the Fock matrix elements, and of the case (I-PN), where all the Fock matrix elements are small. This relationship (eqn (16)) seems to hold certainly in the cases (I-PP), (I-PN) and (IIPP) investigated here. The inequality eqn (16) indicates that TTA is much easier to achieve its energetic requirement than SF in covalently-linked dimers. At the same time, it is also shown that our strategy of using multiple bridges, which breaks eqn (16), is a powerful tool for realizing SF exothermically. Indeed, this has been achieved in the case (II-PN), where only the nonhorizontal couplings are enhanced and breaks eqn (16). Eqn (16) seems also consistent with the results of previous SF studies, where most tetracene dimers ${ }^{\mathbf{4 1 , 4 2 , 4 4 - 4 6}}$ and crystals ${ }^{15,18-20,22,25,26}$ show the endothermic behaviours except for BET-B. ${ }^{49}$

Finally, we examine the above perturbation analysis through the full diagonalization of the Hamiltonian eqn (1), see the $\mathrm{ESI}, \dagger$ where the relative eigenenergies and main characters of the eigenstates are presented (Fig. S9 and S10 $\dagger$ ). We find good agreement between the perturbative analysis and the full diagonalization in both the energies and characters in the cases of odd combinations of $\mathbf{1 -}(\boldsymbol{m}, \boldsymbol{n})$, and of odd and even combinations of $2-(\boldsymbol{m}, \boldsymbol{n})$. However, in the result of full diagonalization, we also find that the even combinations of $1-(m, n)$ have the lowest excited eigenstates mainly composed of TT character, which are different from those predicted by the perturbative analysis. In the even combinations of $1-(m, n)$, all the electronic couplings $F_{i j}$ are large in their magnitudes, and thus they might induce a difficulty in the perturbation approximation. Both FE dominated and TT dominated states are found to be significantly stabilized through the mixing with CT states in these compounds, and thus in such a case a variational calculation may be inevitable. From these results, we conclude that the perturbative approach (eqn (8)) can predict the consistent character of the lowest excited state with that obtained from variational calculations except for the case where both horizontal and non-horizontal couplings are significantly large with similar amplitudes. We also confirm the stabilization of the TT state in $2-(2,1)$ and $2-(3,2)$ is not so much as to slow down SF and as to prohibit the TT state from separating into free triplets. ${ }^{99}$ We have also performed the full diagonalization for BET$\mathrm{B}$, and obtained the lowest eigenstate dominated by TT state $(55.8 \%)$ followed by CT state $(41.8 \%)$. The TT dominated eigenstate was lower in its energy than the next lowest eigenstate dominated by FE character by $647.8 \mathrm{meV}$. Again, we see a qualitative agreement between the results obtained from perturbative and variational approaches.

\subsection{CT state energy dependence}

In the previous section, we discussed the perturbation analysis based on the fixed energy in the CT state, $2.91 \mathrm{eV}, 0.3 \mathrm{eV}$ higher than the FE state. Here, we consider the CT state energy dependence because the CT state energy is known to be sensitive to the environment, which might change the discussion in the previous section. In Fig. 7, we show the effective energy differences $\left(E_{\mathrm{TT}}-E_{\mathrm{FE}}\right)^{\text {eff }}$ in 1- $(\boldsymbol{m}, \boldsymbol{n})$ and $\mathbf{2}-(\boldsymbol{m}, \boldsymbol{n})$ as a function of the relative CT energy $E_{\mathrm{CT}}-E_{\mathrm{FE}}$. Other CT-mediated terms are also shown in the ESI. $\dagger$ We find significant CT energy dependence in all the molecules except for odd combinations of 1-( $\boldsymbol{m}$,
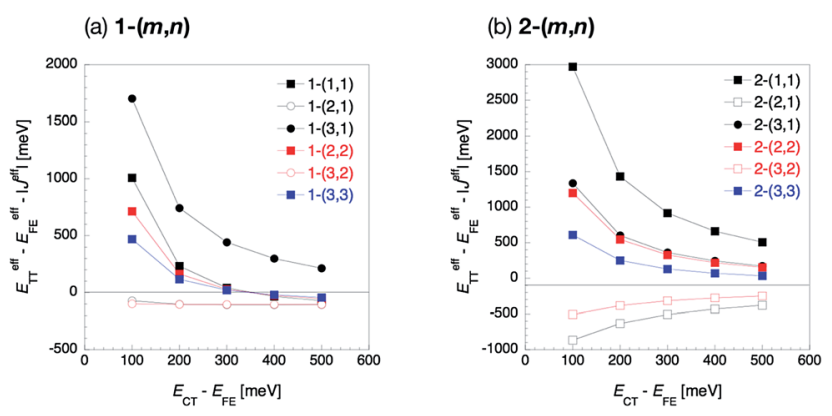

Fig. 7 CT state energy dependence of the effective energy difference defined by eqn (16) of 1-( $m, n)(\mathrm{a})$ and $2-(m, n)(\mathrm{b})$. Filled and blank markers represent even and odd combinations, respectively. Black, red and blue markers represent $n=1,2$ and 3, respectively. Square and circle markers represent $m=n$ and $m \neq n$, respectively. 
$\boldsymbol{n}$ ), where all the Fock matrix elements are small, especially in the small $E_{\mathrm{CT}}$ region. The effective energy difference in the even combinations of $\mathbf{1 -}(\boldsymbol{m}, \boldsymbol{n})$ is found to change from positive to negative values as the relative CT energy $E_{\mathrm{CT}}-E_{\mathrm{FE}}$ goes up from 300 to $400 \mathrm{meV}$. This indicates that the lowest excited state in the even combinations of $\mathbf{1 -}(\boldsymbol{m}, \boldsymbol{n})$ is highly sensitive to the CT state energy, which depends on the environmental condition such as solvent or crystal field effect. This implies that the FECT and TT-CT mixings indicated by large amplitudes of all the Fock matrix elements in this kind of molecules are strong, and thus the effect of the mixing with the CT states is sensitive to the CT state energy. For other molecules, the CT state energy dependence is not serious. This is because, for the odd combinations of $1-(\boldsymbol{m}, \boldsymbol{n})$, all the Fock matrix elements are small, which results in small CT mixing effect and the similar effective energy difference to that of non-mixed states, $E_{\mathrm{TT}}-$ $E_{\mathrm{FE}}$. In contrast, for $\mathbf{2}-(\boldsymbol{m}, \boldsymbol{n})$, the situation is different. In these molecules, either one of the FE energy stabilization originating from the horizontal couplings or TT energy stabilization from the non-horizontal couplings has been found to be significant. Hence, as one of them is large, the relative energy balance between the FE and TT does not likely change even when the CT state energy varies. Consequently, the discussion in the previous section is considered to be weakly dependent on the CT state energy for all the molecules except for the even combinations of $\mathbf{1 -}(\boldsymbol{m}, \boldsymbol{n})$. Finally, we note that the inequality eqn (16) holds for all the CT energy range for the cases (I-PP), (I$\mathrm{PN})$ and (II-PP).

\subsection{Relationship with single-molecular devices and intramolecular electron or hole transfer systems}

We note here the relationships between the above discussion and the previous studies of quantum interference in singlemolecular conductors and intramolecular electron transfer systems, where the quantum interference effect has also been studied. ${ }^{67-69}$ In the research area of single-molecular devices, quantum interference is well recognized and considered as one of the most important issues. In the previous discussions of quantum interference in that field, the phase relationship between a bridge and electrodes, which corresponds to chromophores in this study, do not play any roles in charge conductivity because the electrodes have a continuum state, where we cannot know the phase of wavefunction. The phase effect of an electrode would be averaged and thus be hidden by the dense continuum state, so that the relative sign of couplings provided through each bridge would not affect the total transmission (coupling). However, the relative phase of chromophore MOs at the bridge-linked positions is crucial for SF and TTA, as shown in the comparison between (I-PP) and (II-PP), and between (I-PN) and (II-PN). Due to the discrete energy levels of the HOMO and LUMO of chromophores, we have found crucial roles of the relative phase of the MOs at the bridge-linked positions.

On the other hand, intramolecular electron or hole transfer systems such as norbornyldienes were investigated in terms of electronic coupling through covalent bonds. ${ }^{67,68}$ Many kinds of bridges with different length and connection patterns were considered, and their effects on the electron or hole transfer rate from the quantum interference viewpoint were discussed. Unlike these series of studies, where one of the horizontal couplings is the issue, SF and TTA need an additional consideration of the other electronic coupling, that is, the nonhorizontal coupling, whose relative value to the horizontal one was shown to be crucial to tune the energetic conditions. Consequently, we have found many interesting quantum interference effects in the four cases (I-PP), (I-PN), (II-PP) and (II$\mathrm{PN}$ ) on the FE-TT state energetics, which are not observed in the fields of single-molecular conductors nor intramolecular electron (hole) transfer systems.

\section{Conclusions}

We have investigated the tetracene dimers bridged through two polyynes with various lengths and two bridging patterns as examples of doubly-bridged intramolecular SF and/or TTA systems by density functional theory calculations combined with Green's function method. In order to achieve the desired energetic conditions for SF and/or TTA, we have proposed the idea of quantum interference within a bridge and between bridges both in mathematical and graphical representations. We have discussed the four situations of bridging manners, that is, the cases (I-PP), (I-PN), (II-PP) and (II-PN). The case (I-PP) induces large bridge-mediated contributions in all the Fock matrix elements, while the case (I-PN) induces small bridgemediated contributions in all the Fock matrix elements. The case (II-PP) induces large horizontal couplings and small nonhorizontal couplings, while the case (II-PN) induces small horizontal couplings and large non-horizontal couplings. These four cases are classified by which the PC or NC bridges are used as well as by the bridge-linked positions. As the horizontal and non-horizontal couplings are related to the stabilization in the FE and TT states, respectively, as shown in the perturbation analysis, these element-selective design guidelines of the coupling matrix elements would realize the relative energy tuning for SF or TTA using the same target materials.

Indeed, we have achieved the desired energetic conditions for SF in the cases (I-PN) and (II-PN), and for TTA in the cases (IPP) and (II-PP) using the common chromophore, tetracene. We have analysed the differences of them through the perturbation approach. In the perturbation analysis, we have found that in the case (I-PP) the lowest state is the FE or TT state (CT energy sensitive), in the case (I-PN) the TT state, in the case (II-PP) the FE state and in the case (II-PN) the TT state. In spite of the same lowest state in the cases (I-PN) and (II-PN), we have also found the exothermicity in SF is larger in the case (II-PN) than (I-PN). We have also shown the inequality eqn (16), which represents the importance of the element-selective design of electronic couplings especially for realizing efficient SF in near isothermic chromophores such as tetracene. Comparisons with the previous reports on tetracene dimers indicate the validity of our designed molecules and design strategies for fast and high-yield SF. We have also found that the quantum interference between bridges proposed here is crucial in covalently-linked SF and TTA 
systems in contrast to single molecular conductors and intramolecular electron/hole transfer systems. The proposed strategy for realizing the desired patterns of the electronic couplings in a covalently-linked system is not limited to polyynes but can be applied to many other bridge moieties. Relative MO phases can be easily obtained by conventional $a b$ initio calculations or even by considering a simple orbital diagram derived from, for example, Hückel approximation level of theory.

\section{Acknowledgements}

This work is supported by JSPS KAKENHI Grant Number JPA2645050 in JSPS Research Fellowship for Young Scientists, Grant Number JP25248007 in Scientific Research (A), Grant Number JP24109002 in Scientific Research on Innovative Areas "Stimuli-Responsive Chemical Species", Grant Numbers JP15H00999 and 17H05157 in Scientific Research on Innovative Areas " $\pi$-System Figuration", and Grant Number JP26107004 in Scientific Research on Innovative Areas "Photosynergetics". This is also partly supported by King Khalid University through a grant RCAMS/KKU/001-16 under the Research Center for Advanced Materials Science at King Khalid University, Kingdom of Saudi Arabia.

\section{References}

1 M. B. Smith and J. Michl, Chem. Rev., 2010, 110, 6891-6936.

2 M. B. Smith and J. Michl, Annu. Rev. Phys. Chem., 2013, 64, 361-386.

3 T. N. Singh-Rachford and F. N. Castellano, Coord. Chem. Rev., 2010, 254, 2560-2573.

4 J. Zhou, Q. Liu, W. Feng, Y. Sun and F. Li, Chem. Rev., 2015, 115, 395-465.

5 T. Minami and M. Nakano, J. Phys. Chem. Lett., 2012, 3, 145150.

6 T. Minami, S. Ito and M. Nakano, J. Phys. Chem. Lett., 2012, 3, 2719-2723.

7 T. Minami, S. Ito and M. Nakano, J. Phys. Chem. Lett., 2013, 4, 2133-2137.

8 S. Ito, T. Minami and M. Nakano, J. Phys. Chem. C, 2012, 116, 19729-19736.

9 S. Ito and M. Nakano, J. Phys. Chem. C, 2015, 119, 148-157.

10 S. Ito, T. Nagami and M. Nakano, J. Phys. Chem. Lett., 2016, 7, 3925-3930.

11 T. Zeng and N. Ananth, J. Am. Chem. Soc., 2014, 136, 1263812647.

12 A. Akdag, Z. Havlas and J. Michl, J. Am. Chem. Soc., 2012, 134, 14624-14631.

13 W. M. Moller and M. Pope, J. Chem. Phys., 1973, 59, 27602761.

14 H. Bouchriha, V. Ern, J. L. L. Fave, C. Guthmann and M. Schott, J. Phys., 1978, 39, 257-271.

15 G. B. Piland and C. J. Bardeen, J. Phys. Chem. Lett., 2015, 6, 1841-1846.

16 M. W. B. Wilson, A. Rao, K. Johnson, S. Gélinas, R. Di Pietro, J. Clark and R. H. Friend, J. Am. Chem. Soc., 2013, 135, 1668016688.
17 H. L. Stern, A. J. Musser, S. Gelinas, P. Parkinson, L. M. Herz, M. J. Bruzek, J. Anthony, R. H. Friend and B. J. Walker, Proc. Natl. Acad. Sci. U. S. A., 2015, 201503471.

18 W.-L. Chan, M. Ligges and X.-Y. Zhu, Nat. Chem., 2012, 4, 840-845.

19 S. R. Yost, J. Lee, M. W. B. Wilson, T. Wu, D. P. McMahon, R. R. Parkhurst, N. J. Thompson, D. N. Congreve, A. Rao, K. Johnson, M. Y. Sfeir, M. G. Bawendi, T. M. Swager, R. H. Friend, M. a. Baldo and T. Van Voorhis, Nat. Chem., 2014, 6, 492-497.

20 P. M. Zimmerman, F. Bell, D. Casanova and M. HeadGordon, J. Am. Chem. Soc., 2011, 133, 19944-19952.

21 P. M. Zimmerman, C. B. Musgrave and M. Head-Gordon, Acc. Chem. Res., 2013, 46, 1339-1347.

22 D. Casanova, J. Chem. Theory Comput., 2014, 10, 324-334.

23 X. Feng, A. V. Luzanov and A. I. Krylov, J. Phys. Chem. Lett., 2013, 4, 3845-3852.

24 S. M. Parker and T. Shiozaki, J. Chem. Theory Comput., 2014, 10, 3738-3744.

25 K. Aryanpour, A. Shukla and S. Mazumdar, J. Phys. Chem. C, 2015, 119, 6966-6979.

26 S. M. Parker, T. Seideman, M. A. Ratner and T. Shiozaki, J. Phys. Chem. C, 2014, 118, 12700-12705.

27 B. J. Walker, A. J. Musser, D. Beljonne and R. H. Friend, Nat. Chem., 2013, 5, 1019-1024.

28 M. W. B. Wilson, A. Rao, J. Clark, R. S. S. Kumar, D. Brida, G. Cerullo and R. H. Friend, J. Am. Chem. Soc., 2011, 133, 11830-11833.

29 A. A. Bakulin, S. E. Morgan, T. B. Kehoe, M. W. B. Wilson, A. W. Chin, D. Zigmantas, D. Egorova and A. Rao, Nat. Chem., 2016, 8, 16-23.

30 A. J. Musser, M. Liebel, C. Schnedermann, T. Wende, T. B. Kehoe, A. Rao and P. Kukura, Nat. Phys., 2015, 11, 352-357.

31 L. Wang, Y. Olivier, O. V. Prezhdo and D. Beljonne, J. Phys. Chem. Lett., 2014, 5, 3345-3353.

32 T. C. Berkelbach, M. S. Hybertsen and D. R. Reichman, J. Chem. Phys., 2013, 138, 114103.

33 T. C. Berkelbach, M. S. Hybertsen and D. R. Reichman, J. Chem. Phys., 2014, 141, 074705.

$34 \mathrm{~J} . \mathrm{Li}, \mathrm{Z}$. Chen, Q. Zhang, Z. Xiong and Y. Zhang, Org. Electron., 2015, 26, 213-217.

35 G. B. Piland, J. J. Burdett, D. Kurunthu and C. J. Bardeen, J. Phys. Chem. C, 2013, 117, 1224-1236.

36 S. T. Roberts, R. E. McAnally, J. N. Mastron, D. H. Webber, M. T. Whited, R. L. Brutchey, M. E. Thompson and S. E. Bradforth, J. Am. Chem. Soc., 2012, 134, 6388-6400.

37 J. Herz, T. Buckup, F. Paulus, J. U. Engelhart, U. H. F. Bunz and M. Motzkus, J. Phys. Chem. A, 2015, 119, 6602-6610.

38 S. Amemori, Y. Sasaki, N. Yanai and N. Kimizuka, J. Am. Chem. Soc., 2016, 138, 8702-8705.

39 P. Duan, N. Yanai, H. Nagatomi and N. Kimizuka, J. Am. Chem. Soc., 2015, 137, 1887-1894.

40 D. Kato, H. Sakai, N. V. Tkachenko and T. Hasobe, Angew. Chem., Int. Ed., 2016, 55, 5230-5234.

41 A. M. Müller, Y. S. Avlasevich, K. Müllen and C. J. Bardeen, Chem. Phys. Lett., 2006, 421, 518-522. 
42 A. M. Müller, Y. S. Avlasevich, W. W. Schoeller, K. Müllen and C. J. Bardeen, J. Am. Chem. Soc., 2007, 129, 14240-14250.

43 J. C. Johnson, A. Akdag, M. Zamadar, X. Chen, A. F. Schwerin, I. Paci, M. B. Smith, Z. Havlas, J. R. Miller, M. A. Ratner, A. J. Nozik and J. Michl, J. Phys. Chem. B, 2013, 117, 4680-4695.

44 P. J. Vallett, J. L. Snyder and N. H. Damrauer, J. Phys. Chem. A, 2013, 117, 10824-10838.

45 E. C. Alguire, J. E. Subotnik and N. H. Damrauer, J. Phys. Chem. A, 2015, 119, 299-311.

46 J. D. Cook, T. J. Carey and N. H. Damrauer, J. Phys. Chem. A, 2016, 120, 4473-4481.

47 J. Zirzlmeier, D. Lehnherr, P. B. Coto, E. T. Chernick, R. Casillas, B. S. Basel, M. Thoss, R. R. Tykwinski and D. M. Guldi, Proc. Natl. Acad. Sci. U. S. A., 2015, 112, 53255330.

48 J. Zirzlmeier, R. Casillas, S. R. Reddy, P. B. Coto, D. Lehnherr, E. T. Chernick, I. Papadopoulos, M. Thoss, R. R. Tykwinski and D. M. Guldi, Nanoscale, 2016, 8, 10113-10123.

49 N. V. Korovina, S. Das, Z. Nett, X. Feng, J. Joy, R. Haiges, A. I. Krylov, S. E. Bradforth and M. E. Thompson, J. Am. Chem. Soc., 2016, 138, 617-627.

50 X. Feng, D. Casanova and A. I. Krylov, J. Phys. Chem. C, 2016, 120, 19070-19077.

51 E. G. Fuemmeler, S. N. Sanders, A. B. Pun, E. Kumarasamy, T. Zeng, K. Miyata, M. L. Steigerwald, X.-Y. Zhu, M. Y. Sfeir, L. M. Campos and N. Ananth, ACS Cent. Sci., 2016, 2, 316324.

52 S. Ito, T. Nagami and M. Nakano, J. Phys. Chem. A, 2016, 120, 6236-6241.

53 S. N. Sanders, E. Kumarasamy, A. B. Pun, K. Appavoo, M. L. Steigerwald, L. M. Campos and M. Y. Sfeir, J. Am. Chem. Soc., 2016, 138, 7289-7297.

54 S. N. Sanders, E. Kumarasamy, A. B. Pun, M. T. Trinh, B. Choi, J. Xia, E. J. Taffet, J. Z. Low, J. R. Miller, X. Roy, X. Y. Zhu, M. L. Steigerwald, M. Y. Sfeir and L. M. Campos, J. Am. Chem. Soc., 2015, 137, 8965-8972.

55 S. N. Sanders, E. Kumarasamy, A. B. Pun, M. L. Steigerwald, M. Y. Sfeir and L. M. Campos, Angew. Chem., Int. Ed., 2016, 128, 1-6.

56 T. Zeng and P. Goel, J. Phys. Chem. Lett., 2016, 7, 1351-1358. 57 D. Dzebo, K. Börjesson, V. Gray, K. Moth-Poulsen and B. Albinsson, J. Phys. Chem. C, 2016, 120, 23397-23406.

58 S. Ito, T. Nagami and M. Nakano, Phys. Chem. Chem. Phys., 2017, 19, 5737-5745.

59 J. Vura-Weis, M. D. Newton, M. R. Wasielewski and J. E. Subotnik, J. Phys. Chem. C, 2010, 114, 20449-20460.

60 F. C. Spano, Acc. Chem. Res., 2010, 43, 429-439.

61 N. J. Hestand and F. C. Spano, J. Chem. Phys., 2015, 143, 244707.

62 H. Yamagata, C. M. Pochas and F. C. Spano, J. Phys. Chem. B, 2012, 116, 14494-14503.

63 T. Zeng, J. Phys. Chem. Lett., 2016, 4405-4412.

64 R. Tempelaar and D. R. Reichman, J. Chem. Phys., 2017, 146, 174703.

65 P. C. P. de Andrade and J. A. Freire, J. Chem. Phys., 2003, 118, 6733-6740.
66 P. C. P. de Andrade and J. A. Freire, J. Chem. Phys., 2004, 120, 7811-7819.

67 M. D. Newton, Chem. Rev., 1991, 91, 767-792.

68 K. D. Jordan and M. N. Paddon-Row, Chem. Rev., 1992, 92, 395-410.

69 C. J. Lambert, Chem. Soc. Rev., 2015, 44, 875-888.

70 T. Tada and K. Yoshizawa, Phys. Chem. Chem. Phys., 2015, 17, 32099-32110.

71 Y. Tomkiewicz, R. P. Groff and P. Avakian, J. Chem. Phys., 1971, 54, 4504-4507.

72 J. Tanaka, Bull. Chem. Soc. Jpn., 1964, 38, 86-103.

73 M. Komfort, H. G. Löhmannsröben and T. Salthammer, J. Photochem. Photobiol., A, 1990, 51, 215-227.

74 B. Stevens, S. R. Perez and J. A. Ors, J. Am. Chem. Soc., 1974, 96, 6846-6850.

75 E. J. Bowen, E. Mikiewicz and F. W. Smith, Proc. Phys. Soc., London, Sect. A, 1949, 62, 26-31.

76 K. Tahara and Y. Tobe, Chem. Rev., 2006, 106, 5274-5290.

77 A. Orita and J. Otera, Chem. Rev., 2006, 106, 5387-5412.

78 W. A. Chalifoux and R. R. Tykwinski, C. R. Chim., 2009, 12, 341-358.

79 S. Kato, N. Takahashi and Y. Nakamura, J. Org. Chem., 2013, 78, 7658-7663.

80 Y. Tobe, I. Ohki, M. Sonoda, H. Niino, T. Sato and T. Wakabayashi, J. Am. Chem. Soc., 2003, 125, 5614-5615.

81 I. Hisaki, T. Eda, M. Sonoda, H. Niino, T. Sato, T. Wakabayashi and Y. Tobe, J. Org. Chem., 2005, 70, 18531864.

82 P. N. W. Baxter, A. Al Ouahabi, J. P. Gisselbrecht, L. Brelot and A. Varnek, J. Org. Chem., 2012, 77, 126-142.

83 J. Antony and S. Grimme, Phys. Chem. Chem. Phys., 2006, 8, 5287-5293.

84 H. Iikura, T. Tsuneda, T. Yanai and K. Hirao, J. Chem. Phys., 2001, 115, 3540-3544.

85 C. Lee, W. Yang and R. G. Parr, Phys. Rev. B: Condens. Matter Mater. Phys., 1988, 37, 785-789.

86 Y. Tawada, T. Tsuneda, S. Yanagisawa, T. Yanai and K. Hirao, J. Chem. Phys., 2004, 120, 8425-8433.

87 P. C. Hariharan and J. A. Pople, Theor. Chim. Acta, 1973, 28, 213-222.

88 M. J. Frisch, G. W. Trucks, H. B. Schlegel, G. E. Scuseria, M. A. Robb, J. R. Cheeseman, G. Scalmani, V. Barone, B. Mennucci, G. A. Petersson, H. Nakatsuji, M. Caricato, X. Li, H. P. Hratchian, A. F. Izmaylov, J. Bloino, G. Zheng, J. L. Sonnenberg, M. Hada, M. Ehara, K. Toyota, R. Fukuda, J. Hasegawa, M. Ishida, T. Nakajima, Y. Honda, O. Kitao, H. Nakai, T. Vreven, J. A. Montgomery Jr, J. E. Peralta, F. Ogliaro, M. Bearpark, J. J. Heyd, E. Brothers, K. N. Kudin, V. N. Staroverov, T. Keith, R. Kobayashi, J. Normand, K. Raghavachari, A. Rendell, J. C. Burant, S. S. Iyengar, J. Tomasi, M. Cossi, N. Rega, J. M. Millam, M. Klene, J. E. Knox, J. B. Cross, V. Bakken, C. Adamo, J. Jaramillo, R. Gomperts, R. E. Stratmann, O. Yazyev, A. J. Austin, R. Cammi, C. Pomelli, J. W. Ochterski, R. L. Martin, K. Morokuma, V. G. Zakrzewski, G. A. Voth, P. Salvador, J. J. Dannenberg, S. Dapprich, A. D. Daniels, Ö. Farkas, J. B. Foresman, 
J. V. Ortiz, J. Cioslowski and D. J. Fox, Gaussian 09, Revision B.01, Gaussian, Inc., Wallingford CT, 2009.

89 S. Ito, T. Nagami and M. Nakano, J. Phys. Chem. Lett., 2015, 6, 4972-4977.

90 Y. Yao, Phys. Rev. B, 2016, 93, 1-5.

91 N. Renaud and F. C. Grozema, J. Phys. Chem. Lett., 2015, 6, 360-365.

92 H. Tamura, M. Huix-Rotllant, I. Burghardt, Y. Olivier and D. Beljonne, Phys. Rev. Lett., 2015, 115, 1-5.

93 Y. Fujihashi and A. Ishizaki, J. Phys. Chem. Lett., 2016, 7, 363369.

94 M. Nakano, S. Ito, T. Nagami, Y. Kitagawa and T. Kubo, J. Phys. Chem. C, 2016, 120, 22803-22815.
95 E. Clar, Polyaromatic Hydrocarbons, Academic Press, New York, 1964.

96 G. Weiser, L. Sebastian and F. Physikalische, Chem. Phys., 1981, 61, 125-135.

97 H. Yamagata, J. Norton, E. Hontz, Y. Olivier, D. Beljonne, J. L. Brédas, R. J. Silbey and F. C. Spano, J. Chem. Phys., 2011, 134, 204703.

98 L. Berstis and K. K. Baldridge, Phys. Chem. Chem. Phys., 2015, 17, 30842-30853.

99 E. Busby, T. C. Berkelbach, B. Kumar, A. Chernikov, Y. Zhong, H. Hlaing, X.-Y. Zhu, T. F. Heinz, M. S. Hybertsen, M. Y. Sfeir, D. R. Reichman, C. Nuckolls and O. Yaffe, J. Am. Chem. Soc., 2014, 136, 10654-10660. 